\title{
Forced synchronization of quasiperiodic oscillations in a thermoacoustic system
}

\author{
Yu Guan ${ }^{1}$, Vikrant Gupta ${ }^{2}$, Minping Wan ${ }^{2}$ and Larry K. B. $\mathbf{L i}^{1, \dagger}$ \\ ${ }^{1}$ Department of Mechanical and Aerospace Engineering, The Hong Kong University of Science \\ and Technology, Clear Water Bay, Hong Kong \\ ${ }^{2}$ Department of Mechanics and Aerospace Engineering, Southern University of Science \\ and Technology, Shenzhen, China
}

(Received 23 March 2019; revised 13 August 2019; accepted 13 August 2019;

first published online 27 September 2019)

In self-excited combustion systems, the application of open-loop forcing is known to be an effective strategy for controlling periodic thermoacoustic oscillations, but it is not known whether and under what conditions such a strategy would work on thermoacoustic oscillations that are not simply periodic. In this study, we experimentally examine the effect of periodic acoustic forcing on a prototypical thermoacoustic system consisting of a ducted laminar premixed flame oscillating quasiperiodically on an ergodic $\mathbb{T}^{2}$ torus at two incommensurate natural frequencies, $f_{1}$ and $f_{2}$. Compared with that of a classical period- 1 system, complete synchronization of this $\mathbb{T}_{1,2}^{2}$ system is found to occur via a more intricate route involving three sequential steps: as the forcing amplitude, $\epsilon_{f}$, increases at a fixed forcing frequency, $f_{f}$, the system transitions first (i) to ergodic $\mathbb{T}_{1,2, f}^{3}$ quasiperiodicity; then (ii) to resonant $\mathbb{T}_{1, f}^{2}$ quasiperiodicity as the weaker of the two natural modes, $f_{2}$, synchronizes first, leading to partial synchronization; and finally (iii) to a $P 1_{f}$ limit cycle as the remaining natural mode, $f_{1}$, also synchronizes, leading to complete synchronization. The minimum $\epsilon_{f}$ required for partial and complete synchronization decreases as $f_{f}$ approaches either $f_{1}$ or $f_{2}$, resulting in two primary Arnold tongues. However, when forced at an amplitude above that required for complete synchronization, the system can transition out of $P 1_{f}$ and into $\mathbb{T}_{1,2, f}^{3}$ or $\mathbb{T}_{2, f}^{2}$. The optimal control strategy is to apply off-resonance forcing at a frequency around the weaker natural mode $\left(f_{2}\right)$ and at an amplitude just sufficient to cause $P 1_{f}$, because this produces the largest reduction in thermoacoustic amplitude via asynchronous quenching. Analysis of the Rayleigh index shows that this reduction is physically caused by a disruption of the positive coupling between the unsteady heat release rate of the flame and the $f_{1}$ and $f_{2}$ acoustic modes. If the forcing is applied near the stronger natural mode $\left(f_{1}\right)$, however, resonant amplification can occur. We then phenomenologically model this $\mathbb{T}_{1,2}^{2}$ thermoacoustic system as two reactively coupled van der Pol oscillators subjected to external sinusoidal forcing, and find that many of its synchronization features - such as the three-step route to $P 1_{f}$, the double Arnold tongues, asynchronous quenching and resonant amplification - can be qualitatively reproduced. This shows that these features are not limited to our particular system, but are universal features of forced self-excited oscillators. 
This study extends the applicability of open-loop control from classical period-1 systems with just a single time scale to ergodic $\mathbb{T}^{2}$ quasiperiodic systems with two incommensurate time scales.

Key words: instability control, nonlinear instability

\section{Introduction}

Increasingly stringent emissions regulations have prompted gas turbine manufacturers to switch to lean premixed combustion, but doing so provokes thermoacoustic instability (Lieuwen \& Yang 2005). This phenomenon arises from positive coupling between the heat-release-rate (HRR) oscillations of an unsteady flame and one or more of the natural acoustic modes of the combustion chamber (Culick 2006). If the HRR oscillations are sufficiently in phase with the acoustic pressure oscillations, the former can transfer energy to the latter via the Rayleigh (1878) mechanism, resulting in high-amplitude self-excited flow oscillations at the characteristic acoustic frequencies of the system (Candel 2002). If left unchecked, such thermoacoustic oscillations can reduce flame stability and increase thermal stresses (Lieuwen \& Yang 2005). It is thus important to be able to control thermoacoustic oscillations in combustion systems (Poinsot 2017).

\subsection{Quasiperiodicity in self-excited thermoacoustic systems}

Both passive and active methods are available to control thermoacoustic oscillations (Candel 2002; Lieuwen \& Yang 2005), but most assume a priori that such oscillations are periodic with a single characteristic frequency and a time-independent amplitude, i.e. they assume period-1 limit cycles (Kashinath, Li \& Juniper 2018). This assumption, however, is not always valid (Juniper \& Sujith 2018). Even early on, time-domain simulations by Jahnke \& Culick (1994) have shown that a combustor with multiple acoustic modes can undergo a Neimark-Sacker (torus-birth) bifurcation to quasiperiodicity. In nonlinear dynamical systems, quasiperiodicity is a common state arising from interactions between at least two periodic modes whose natural frequencies, $f_{1}$ and $f_{2}$, are incommensurate, i.e. their winding number, $f_{2} / f_{1}$, is irrational. A two-frequency quasiperiodic system thus oscillates with a period of infinity and evolves in phase space along a non-repeating orbit on a $\mathbb{T}^{2}$ (two-dimensional) torus attractor formed by modes at $f_{1}$ and $f_{2}$ (Thompson \& Stewart 2002).

Those early simulations by Jahnke \& Culick (1994) have since been joined by numerous laboratory experiments showing similarly aperiodic dynamics. For example, Kabiraj et al. (2012a), Kabiraj, Sujith \& Wahi (2012b) and Kabiraj \& Sujith (2012) have shown that even a simple thermoacoustic system (a tube containing laminar premixed flames) can undergo a bifurcation cascade as a control parameter (the flame position within the tube) is varied, producing not just period-1 limit cycles, but also period- $k$, frequency-locked, intermittent, chaotic and quasiperiodic oscillations. Later, Kashinath, Waugh \& Juniper (2014) were able to reproduce these experimental findings, including quasiperiodicity, using low-order simulations of a $G$-equation-based laminar premixed flame coupled with Galerkin acoustics. This has been followed by further experimental evidence of quasiperiodicity in both laminar (Vishnu, Sujith \& 
Aghalayam 2015) and turbulent (Gotoda et al. 2015) systems. However, despite this growing evidence of quasiperiodic oscillations appearing in a variety of self-excited thermoacoustic systems, the control and suppression of such oscillations remain largely unexplored.

\subsection{Forced synchronization of periodic oscillations}

Unlike quasiperiodic oscillations, periodic oscillations have been the subject of various control strategies in thermoacoustics, ranging from passive methods (Lieuwen 2003; Noiray et al. 2009) to active methods (Heckl 1988; Dowling \& Morgans 2005; Bothien, Moeck \& Paschereit 2008). The simplest form of the latter is open-loop control, which requires only a single actuator (e.g. a fuel solenoid valve or a loudspeaker; McManus, Vandsburger \& Bowman 1990; Lubarsky et al. 2003) and no sensors or feedback controllers, whose reliability can be questionable under the severe operating conditions of most combustors (Mongia et al. 2003).

A versatile way to study open-loop control is in the framework of forced synchronization (Pikovsky, Rosenblum \& Kurths 2003). In forced synchronization, a self-excited system oscillating at one or more of its natural frequencies is externally forced to oscillate at a different frequency (Balanov et al. 2008). This nonlinear process has been studied in many natural and technological systems (e.g. circadian rhythms and optical lasers; Glass 2001; Boccaletti et al. 2002) and has been modelled with low-order universal oscillators such as the forced van der Pol (1927) (VDP) oscillator. The use of open-loop forcing to control self-excited oscillations has been gaining attention owing to its potential application in fields as diverse as optoelectronics, psychophysics and hydrodynamics (Hovel 2010).

In thermoacoustics, open-loop forcing has been shown to be able to weaken periodic self-excited oscillations in various combustion systems, ranging from the simple Rijke tube (Reynolds numbers of $R e \sim 10^{3}$ with natural frequencies of $f_{1} \sim 10^{2} \mathrm{~Hz}$; Guan, Murugesan \& Li 2018; Kashinath et al. 2018; Guan et al. 2019a; Mondal, Pawar \& Sujith 2019) to turbulent premixed combustors $\left(R e \sim 10^{4}, f_{1} \sim 10^{2} \mathrm{~Hz}\right.$; Bellows, Hreiz \& Lieuwen 2008; Balusamy et al. 2015). A recurring theme of these studies has been the application of periodic acoustic forcing to periodic thermoacoustic oscillations, accompanied by an examination of the nonlinear dynamics en route to and beyond the synchronization boundaries. For example, on applying periodic acoustic forcing at an off-resonance frequency to a periodically oscillating laminar premixed flame in a tube, Guan et al. (2019a) found: (i) a transition from unforced periodicity to $\mathbb{T}^{2}$ quasiperiodicity via a Neimark-Sacker bifurcation; (ii) a subsequent transition from $\mathbb{T}^{2}$ quasiperiodicity to $1: 1$ synchronization at a critical forcing amplitude; (iii) a $\vee$-shaped Arnold tongue centred on the natural frequency; (iv) two different routes to synchronization, one via a saddle-node bifurcation and one via an inverse Neimark-Sacker bifurcation; and (v) that all of these dynamics could be qualitatively reproduced with a low-order universal oscillator containing a VDP kernel. Balusamy et al. (2015) applied similar off-resonance forcing to a swirl-stabilized turbulent premixed combustor and found additional synchronization dynamics, such as frequency pushing and pulling as well as phase locking, slipping, drifting and trapping - the latter a partially synchronous feature defined by frequency locking without phase locking (Li \& Juniper 2013c). For high forcing amplitudes, Guan et al. (2018) found that a synchronized system can transition out of synchronization and into strange non-chaotic and chaotic states, behaving in line with the Afraimovich \& Shilnikov (1991) theorem for the collapse of a phase-locked torus. 
From a control perspective, numerous studies have shown that, near the onset of synchronization, the thermoacoustic amplitude can be drastically reduced - typically to less than $20 \%$ of that of the unforced state (Bellows et al. 2008; Kashinath et al. 2018; Guan et al. 2019a,b; Mondal et al. 2019). This reduction occurs via asynchronous quenching, a nonlinear process in which the amplitude of a self-excited oscillator is reduced by the external application of periodic forcing at a frequency far enough from the natural frequency to prevent resonant amplification of the forcing signal (Minorsky 1967). Asynchronous quenching can be modelled with a forced VDP oscillator (Dewan 1972) and has been exploited for open-loop control in various fields, ranging from plasma physics (Keen \& Fletcher 1970) to hydrodynamics (Staubli 1987). In thermoacoustics, asynchronous quenching has recently been shown to coincide with an inverse Neimark-Sacker bifurcation to synchronization and a reduced Rayleigh index (Guan et al. 2019a; Mondal et al. 2019). However, it is not known how effective this control strategy would be if it were applied to thermoacoustic oscillations that are quasiperiodic rather than periodic. Specifically, it is not known how the strategy would have to be modified to cope with the presence of an additional (second) natural mode. For example, in the periodic case, the thermoacoustic amplitude can be reduced by applying forcing at a frequency sufficiently far from the natural frequency for asynchronous quenching to occur, as demonstrated by Guan et al. (2019a) and Mondal et al. (2019). However, if a second natural mode is present, its frequency could coincide with the forcing frequency, resulting in resonant amplification (Minorsky 1967). A potential way to avoid such amplification is to ensure that the forcing frequency is sufficiently far from both natural frequencies for asynchronous quenching to occur in both natural modes. However, this assumes that the two natural modes are similar in strength, which is not always the case in practice. As we will see in $\S 3$, when one natural mode is significantly weaker than the other, it is possible to reduce the overall thermoacoustic amplitude by applying forcing at a frequency around the weaker natural mode and exploiting the same suppression mechanism (asynchronous quenching) as in the periodic case, albeit in more steps.

\subsection{Forced synchronization of quasiperiodic oscillations}

Two primary types of phase trajectories can exist on a two-frequency quasiperiodic attractor: a resonant limit cycle or an ergodic $\mathbb{T}^{2}$ torus (Balanov et al. 2008). The former arises when the two natural modes are mutually synchronized such that their frequencies are commensurate, resulting in a phase trajectory that evolves along a closed periodic orbit on the quasiperiodic attractor. The latter arises when the two natural modes are not mutually synchronized and hence their frequencies are not commensurate, resulting in a phase trajectory that spirals non-repeatedly through every point on the quasiperiodic attractor. In this study, we focus on the forced synchronization of the latter type of solution. However, both types are reviewed here because they are closely related to each other.

Anishchenko, Nikolaev \& Kurths $(2007,2008)$ have examined the effect of external forcing on a resonant limit cycle residing on a $\mathbb{T}^{2}$ torus. They used both (i) numerical simulations of an autonomous quasiperiodic oscillator - which was modelled as two coupled VDP oscillators - with two natural modes, $f_{1}$ and $f_{2}$, undergoing mutual synchronization and subjected to sinusoidal forcing and (ii) laboratory experiments on an electronic circuit representing that oscillator. Those researchers found that the resonant limit cycle could generally be synchronized with the forcing. By analysing 
their data with Lyapunov exponents and Poincaré maps, they were able to identify the following four distinct states as the forcing amplitude increased at an off-resonance frequency, $f_{f}$ (figures 5 and 6 of Anishchenko et al. 2008).

(A) The $f_{f}$ mode is introduced, but mutual synchronization of $f_{1}$ and $f_{2}$ continues, causing the resonant limit cycle to give way to a resonant $\mathbb{T}_{f, 1=2}^{2}$ torus at $f_{f}$ and $f_{1}=f_{2}$.

(B) Mutual synchronization no longer occurs, allowing $f_{1}$ and $f_{2}$ to assume different values, causing the resonant $\mathbb{T}_{f, 1=2}^{2}$ torus to give way to an ergodic $\mathbb{T}_{f, 1,2}^{3}$ torus with three incommensurate modes: $\hat{f}_{f}, f_{1}$ and $f_{2}$. As $f_{f}$ varies, different partial resonances can occur on the $\mathbb{T}_{f, 1,2}^{3}$ torus, leading to other $\mathbb{T}^{2}$ quasiperiodic and chaotic states (Stankevich, Kurths \& Kuznetsov 2015).

(C) Partial synchronization occurs, in which one of the natural modes synchronizes with the forcing $\left(f_{2}=f_{f}\right)$, while the other remains desynchronized $\left(f_{1} \neq f_{f}\right)$. This leads to the emergence of a resonant $\mathbb{T}_{f=2,1}^{2}$ torus on the existing $\mathbb{T}_{f, 1,2}^{3}$ torus.

(D) Complete synchronization occurs, in which both natural modes synchronize with the forcing $\left(f_{f}=f_{1}=f_{2}\right)$, producing a period-1 limit cycle at $f_{f}$, as denoted by $P 1_{f}$. In the literature, the term 'complete synchronization' is sometimes used to refer to the suppression of signal differences between two or more identical coupled chaotic oscillators (Pikovsky et al. 2003). In this study, however, we follow the convention of Anishchenko et al. (2008) by using this term to refer to the forced synchronization of the natural modes of a $\mathbb{T}^{2}$ quasiperiodic oscillator.

As mentioned earlier, a quasiperiodic attractor can also host an ergodic $\mathbb{T}^{2}$ torus. Loose, Wünsche \& Henneberger (2010) have experimentally investigated the forced synchronization of such a torus using semiconductor lasers. They found similar states to those reported by Anishchenko et al. $(2007,2008)$ (see above), with the exception that state (A) was bypassed because the two natural modes were not mutually synchronized at zero or low forcing amplitudes. Crucially, they also validated the VDP-oscillator predictions from Anishchenko et al. (2007, 2008), showing that weak internal coupling leads to the partial synchronization of only one natural mode, but that strong internal coupling leads to the complete synchronization of both natural modes in succession. This qualitative agreement between complex experiments and simple phenomenological modelling provides compelling arguments for the universality of such synchronization phenomena (Loose et al. 2010).

In thermoacoustics, only one study has previously examined the forced synchronization of an ergodic $\mathbb{T}^{2}$ torus. Using low-order simulations of a ducted laminar premixed flame, Kashinath et al. (2018) showed that applying periodic acoustic forcing at the dominant frequency of an ergodic $\mathbb{T}^{2}$ torus can cause it to completely synchronize with the forcing. However, because only one resonant value of $f_{f}$ was used, neither the partial/complete synchronization boundaries nor the regimes of asynchronous quenching could be explored.

\subsection{Contributions of this study}

In this study, we take a synchronization approach to answering three research questions on the open-loop control of ergodic $\mathbb{T}^{2}$ quasiperiodic thermoacoustic oscillations.

(i) Previous studies have shown that periodic acoustic forcing can control periodic thermoacoustic oscillations $(\S 1.2)$, but can it also control quasiperiodic thermoacoustic oscillations? If it can, how does the synchronization process differ from that of the classical period-1 case studied by Guan et al. (2019a) and Mondal et al. (2019)? 
(ii) If periodic acoustic forcing can control quasiperiodic thermoacoustic oscillations, what are the optimal forcing conditions for producing the maximum reduction in thermoacoustic amplitude? It is necessary to know this if one is to fully exploit synchronization phenomena, such as asynchronous quenching (§ 1.2), for open-loop control.

(iii) Previous studies on electronic circuits and semiconductor lasers have shown that the forced synchronization of quasiperiodic oscillations can be qualitatively modelled with low-order universal oscillators containing a VDP kernel (§1.3), but can such a phenomenological modelling approach work on thermoacoustic systems as well? In other words, can the forced synchronization of quasiperiodic thermoacoustic oscillations - particularly the partial/complete synchronization boundaries and asynchronous quenching - be qualitatively modelled with just two coupled VDP oscillators forced by a sinusoidal term? If so, this would strengthen the universality of synchronization in physically disparate systems and open up new possibilities for the development of active control strategies in thermoacoustic systems with multiple time scales.

To answer these questions, we perform experiments on a prototypical thermoacoustic system (\$2) that is known to exhibit a variety of nonlinear self-excited states, including ergodic $\mathbb{T}^{2}$ quasiperiodicity. We acoustically force this system around its two natural frequencies, at varying amplitudes, and measure its pressure and HRR response. By analysing the data within a synchronization framework, we find that the forcing can weaken $\mathbb{T}^{2}$ quasiperiodic thermoacoustic oscillations - via the sequential birth of partially and completely synchronous states - if the forcing frequency and amplitude are appropriately chosen $(\S 3)$. We then show that these dynamics can be qualitatively reproduced with two coupled VDP oscillators forced by a sinusoidal term $(\S 4)$. We conclude this paper by discussing the implications and limitations of these findings $(\S 5)$.

\section{Experimental set-up and data analysis}

The prototypical thermoacoustic system under study consists of a laminar conical premixed flame in a tube combustor subjected to external acoustic forcing. Figure 1 shows the experimental set-up, which is identical to that of our recent studies on periodic thermoacoustic oscillations (Guan et al. 2018, 2019a,b) and which is modelled after the numerical set-up of Kashinath et al. (2018). The system has four main components: a stainless steel burner (inner diameter, ID: $16.8 \mathrm{~mm}$; length: $800 \mathrm{~mm}$ ), a quartz tube combustor with double open ends (ID: $44 \mathrm{~mm}$; length: $L=860 \mathrm{~mm}$ ), an acoustic decoupler (ID: $180 \mathrm{~mm}$; length: $200 \mathrm{~mm}$ ) and a loudspeaker for acoustic forcing. A copper extension tip (ID: $D=12 \mathrm{~mm}$; length: $30 \mathrm{~mm}$ ) containing a fine-mesh screen is mounted at the burner exit for improved flame stability. The flame is created from a premixed mixture of air and liquefied petroleum gas (LPG: $70 \%$ butane, $30 \%$ propane). The flow rate of the LPG is metered with a rotameter $( \pm 2.5 \%)$, while that of the air is metered with a mass flow controller (Alicat MCR Series: $\pm 0.2 \%$ ). The two reactants are then brought together in a mixing chamber before being piped to the burner.

When unforced, this thermoacoustic system can exhibit a variety of nonlinear self-excited states - including period-1 limit cycles, quasiperiodicity and chaos depending on the equivalence ratio and the flame position within the combustor (Guan et al. 2018, 2019a,b). As this study focuses on the open-loop control of ergodic $\mathbb{T}^{2}$ quasiperiodic oscillations $(\S 1.4)$, we apply forcing to a quasiperiodic state 


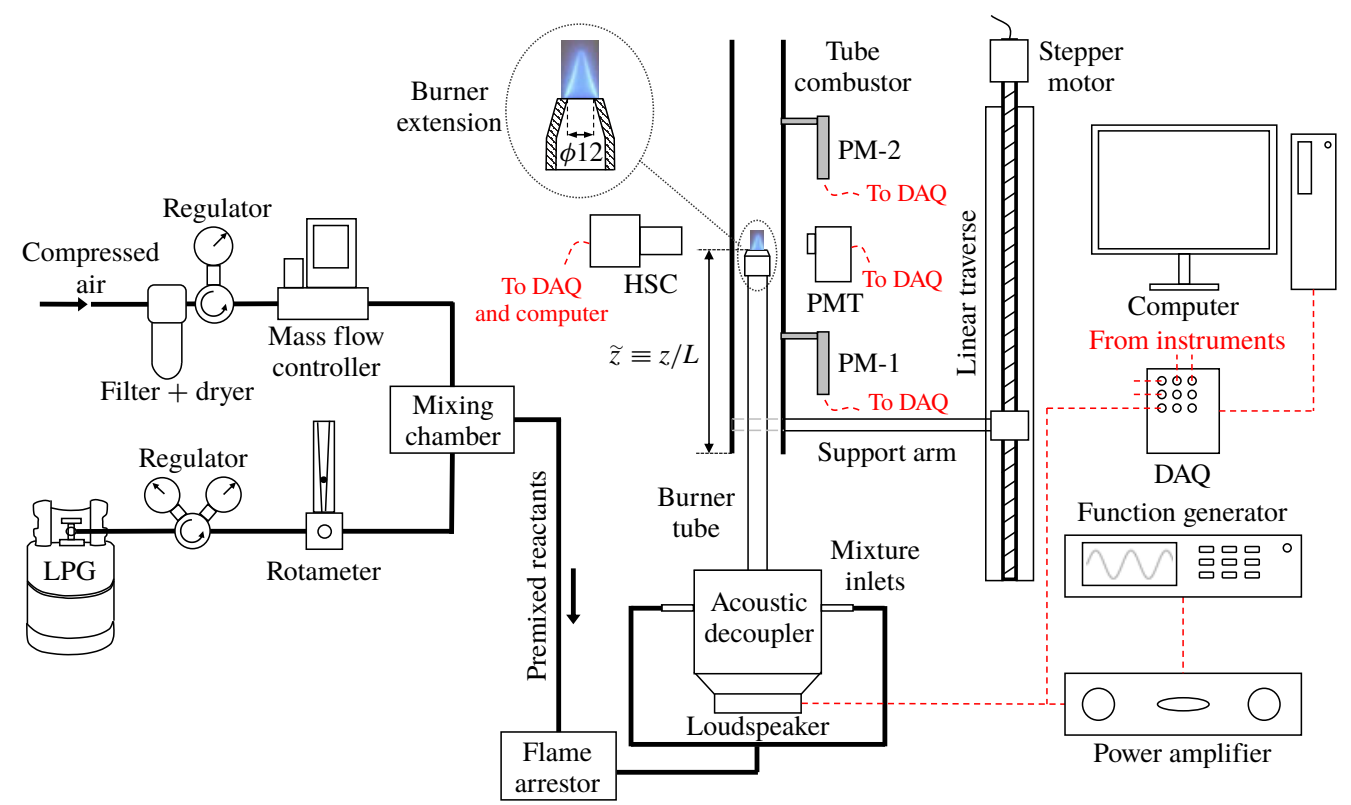

FIgURE 1. (Colour online) Diagram of the experimental set-up, whose main components include a stainless steel burner, a copper burner extension (see inset), a quartz tube combustor with double open ends, an acoustic decoupler, a loudspeaker and a motorized linear traverse for adjusting the flame position $(\tilde{z} \equiv z / L)$ within the combustor. The key dimensions of these components are stated in the text. The measurement diagnostics include two probe microphones (PM-1, PM-2) for the acoustic pressure in the combustor, a hot-wire probe (not shown) for the acoustic velocity perturbation at the burner exit, a photomultiplier tube (PMT) for the global $\mathrm{CH}^{*}$ chemiluminescence from the flame and a high-speed camera (HSC) for time-resolved flame imaging. All dimensions shown are in millimetres. The diagram is not drawn to scale.

containing two incommensurate natural frequencies: $f_{1}$ and $f_{2}$. The details of this state will be examined in $\$ 3.1$.

To generate the forcing, we use a loudspeaker (FaitalPRO 6FE100) mounted in the acoustic decoupler (figure 1) and driven by a power amplifier (Alesis RA150) controlled by a digital function generator (Keysight 33512B) with a sinusoidal output. We apply the forcing across a range of amplitudes (up to flame blow-off) and frequencies $\left(0.60 \leqslant f_{f} / f_{1} \leqslant 1.20 ; 0.86 \leqslant f_{f} / f_{2} \leqslant 1.71\right)$ so as to explore the full synchronization dynamics around both $f_{1}$ and $f_{2}$. We define the forcing amplitude as the velocity perturbation amplitude at the burner exit normalized by the time-averaged velocity of the bulk reactants: $\epsilon_{f} \equiv\left|u^{\prime}\right| / \bar{u}$. We measure $\left|u^{\prime}\right|$ with a hot-wire anemometer (Dantec MiniCTA with a $5 \mu \mathrm{m}$ diameter tungsten probe) operating in constant temperature mode and calibrated to an uncertainty of $\pm 1.3 \%$ via the procedure of Johnson, Uddin \& Pollard (2005).

To characterize the synchronization dynamics and compute the Rayleigh index, we use simultaneous measurements of the acoustic pressure fluctuations in the combustor $\left(p^{\prime}\right)$ and of the global HRR fluctuations from the flame $\left(q^{\prime}\right)$. We measure $p^{\prime}(t)$ with two probe microphones (GRAS 40SA: sensitivity of $3 \mathrm{mV} \mathrm{Pa}^{-1} ; \pm 2.5 \times 10^{-5} \mathrm{~Pa}$ ) mounted $43 \mathrm{~mm}$ (PM-1) and $387 \mathrm{~mm}$ (PM-2) from the bottom of the combustor (figure 1). In most of our analyses, however, we use the $p^{\prime}(t)$ signal from PM-2 
because it behaves qualitatively similarly to that from PM-1 but has a higher signal-to-noise ratio and is located exactly at the flame position, which is important for computing the Rayleigh index. Before each test run, we calibrate both microphones against a certified sound source (Brüel \& Kjær Type 4231). We measure $q^{\prime}(t)$ with a photomultiplier tube (PMT: Thorlabs PMM01; $\pm 1.5 \%$ ) viewing through a bandpass optical filter centred on $430 \mathrm{~nm}$ (10 nm bandwidth), thus capturing the $\mathrm{CH}^{*}$ chemiluminescence emission from the flame (Gaydon 1974). At each forcing condition, we digitize the voltage signals from the microphones and PMT at $16384 \mathrm{~Hz}$ for $6 \mathrm{~s}$ on a 16-bit data acquisition system (DAQ: NI USB-6356).

We process the data in several ways, including (i) in the frequency domain via the power spectral density (PSD), which is computed using the Welch (1967) algorithm, with Hamming windows to reduce spectral leakage, resulting in a frequency resolution of $0.5 \mathrm{~Hz}$; (ii) via the complex analytic signal, which is computed with the Hilbert transform (Gabor 1946) and is used to determine the instantaneous phase difference between the forcing and the system, $\Delta \psi_{f, p^{\prime}} \equiv \psi_{f}-\psi_{p^{\prime}}$; and (iii) in phase space via nonlinear time-series analysis (Kantz \& Schreiber 2003). It should be cautioned that, when applied to an aperiodic signal with multiple different frequencies, the Hilbert transform can give physically undefined values of the instantaneous phase (Boashash 1992). This limitation, however, does not necessarily imply that the Hilbert transform cannot still provide useful insight into the forced synchronization of a system oscillating aperiodically (Pikovsky et al. 1997; Mondal, Pawar \& Sujith 2017). In this study, we follow the convention of the synchronization community by taking a conservative approach whereby the results of the Hilbert transform are cross-checked against other independent synchronization indicators. For example, in nonlinear time-series analysis, we reconstruct the phase space using the embedding theorem of Takens (1981). In this procedure, we determine (i) the optimal time delay $(\tau)$ via the first local minimum of the average mutual information function (Fraser \& Swinney 1986) and (ii) the minimum embedding dimension $(d)$ via the method of Cao (1997). After reconstructing the phase space, we visualize the attractor (limit set) within it using three-dimensional phase portraits and one-sided Poincaré maps. To quantify the degree of topological self-similarity in the attractor, we compute its correlation dimension $\left(\overline{D_{c}}\right)$ with the algorithm of Grassberger \& Procaccia (1983). This is an invariant measure of the number of active degrees of freedom (DOFs) in a dynamical system and can be used to distinguish between a fixed point $\left(\overline{D_{c}}=0\right)$, a periodic limit cycle $\left(\overline{D_{c}}=1\right)$, a $\mathbb{T}^{2}$ torus $\left(\overline{D_{c}}=2\right)$, a $\mathbb{T}^{3}$ torus $\left(\overline{D_{c}}=3\right)$ and a strange attractor $\left(\overline{D_{c}}=\right.$ non-integer $)$. For a detailed discussion of these methods, the reader is referred to the books by Kantz \& Schreiber (2003) and Small (2005).

To complement the $p^{\prime}(t)$ and $q^{\prime}(t)$ data, we capture time-resolved broadband chemiluminescence images of the flame with a high-speed camera (Phantom M310: dynamic range of 12 bits) operating at an image resolution of $512 \times 512$ pixels and a frame rate of $3000 \mathrm{~Hz}$. As $\$ 3.1$ will show, this frame rate is an order of magnitude higher than the highest natural frequency of the self-excited $\mathbb{T}^{2}$ thermoacoustic oscillations $\left(f_{1}=248 \pm 1.5 \mathrm{~Hz}\right)$, ensuring that the flame imaging is time resolved.

\section{Experimental results and discussion}

\subsection{Natural self-excited dynamics: ergodic $\mathbb{T}^{2}$ quasiperiodicity}

Before examining the forced dynamics, it is necessary to find an unforced self-excited state with ergodic $\mathbb{T}^{2}$ quasiperiodicity at two incommensurate frequencies. In our system, such a state can be found at an equivalence ratio of $\phi=0.57( \pm 3.2 \%)$, 


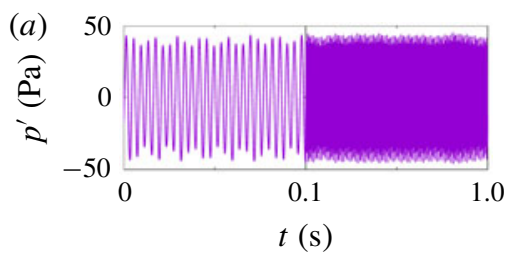

(c)

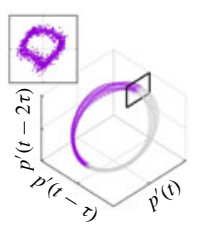

$(d)$

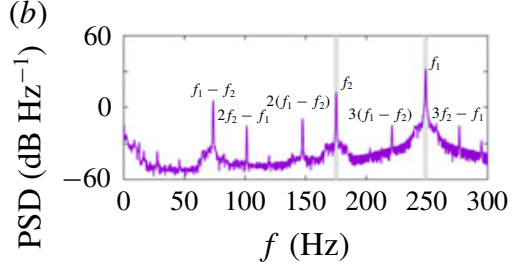

$(e)$

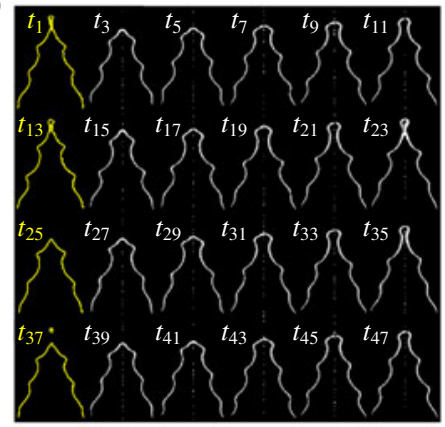

FIgURE 2. (Colour online) Natural self-excited dynamics of a thermoacoustic system undergoing ergodic $\mathbb{T}^{2}$ quasiperiodic oscillations: (a) time trace, (b) PSD, (c) phase portrait and Poincaré map and $(d)$ slope of the correlation sum, all computed from the acoustic pressure fluctuations ( $p^{\prime}$ from PM-2) in the combustor. In $(c)$, phase-space reconstruction is performed with $d=3$ and $\tau=0.005 \mathrm{~s}$. Panel $(e)$ shows a sequence of time-resolved Abel-inverted images of the flame captured via broadband chemiluminescence (successive images are separated in time by $1 / 1500 \mathrm{~s}$ ).

a bulk reactant velocity of $\bar{u}=1.58 \mathrm{~m} \mathrm{~s}^{-1}( \pm 0.2 \%)$, a Reynolds number of $R e \equiv$ $\rho \bar{u} D / \mu=1280( \pm 1.7 \%)$, where $\rho$ and $\mu$ are the density and dynamic viscosity of the reactants, and a flame position of $\tilde{z} \equiv z / L=0.45( \pm 0.4 \%)$, where $z$ is the distance between the burner extension tip and the bottom of the combustor (figure 1). To consolidate the discussion, we present results mostly from this operating condition, but note that its synchronization dynamics (e.g. its bifurcations, synchronous states and Arnold tongues) are qualitatively representative of a range of operating conditions in which $\mathbb{T}^{2}$ quasiperiodicity occurs.

Figure 2 shows the natural self-excited dynamics at this operating condition. To establish the existence of ergodic $\mathbb{T}^{2}$ quasiperiodicity, we examine a variety of indicators, beginning with the $(a)$ time trace, $(b)$ PSD and $(c)$ phase portrait and Poincare map of the acoustic pressure fluctuations ( $p^{\prime}$ from PM-2) in the combustor. The first indication that the system is quasiperiodic can be found in the PSD (figure $2 b$ ), where a pair of sharp dominant peaks appears at two incommensurate natural frequencies: $f_{1}=248 \pm 1.5$ and $f_{2}=174 \pm 1.5 \mathrm{~Hz}$. The peak at $f_{1}$ is nearly $20 \mathrm{~dB}$ (i.e. 10 times) higher than that at $f_{2}$, which, as we will see in $\$ 3.2 .3$, has fundamental implications for the way in which the system transitions from partial to complete synchronization. Surrounding these two peaks $\left(f_{1}\right.$ and $\left.f_{2}\right)$ are several weaker peaks at their linear combinations $\left(\left|n f_{1} \pm m f_{2}\right|\right.$, where $n$ and $m$ are integers), indicating the presence of nonlinear wave-triad interactions between the two natural modes (Schmid \& Henningson 2012). In the time trace (figure 2a), these interactions give rise to amplitude modulations at a relatively low frequency of $f=f_{1}-f_{2} \approx 74 \mathrm{~Hz}$. These beating modulations are a classical feature of self-excited oscillators with multiple natural modes (Pikovsky et al. 2003) and have been observed in various quasiperiodic systems, including flame-driven combustors (Mondal et al. 2017). The quasiperiodic nature of this system is also evident in its phase portrait (figure $2 c$ ), where the phase trajectory can be seen evolving on a stable attractor with a toroidal topology, i.e. a torus attractor. In the Poincaré map (inset of figure $2 c$ ), the intercepts 
of this trajectory form a closed continuous ring, indicating that the torus is ergodic with an irrational winding number (Hilborn 2000).

To verify the two-dimensionality of the torus, we estimate its fractal dimension by computing $\overline{D_{c}}$ as per $\S 2$. Figure $2(d)$ shows the local slope of the correlation sum $\left(D_{c}=\partial \log C_{N} / \partial \log R\right)$ as a function of the normalized hypersphere radius $\left(R / R_{\max }\right)$ for three embedding dimensions $\left(d=8,10\right.$ and 12), all of which are high enough for $D_{c}$ to converge. Within the self-similar Euclidean scaling range $\left(10^{-2} \leqslant R / R_{\max } \leqslant 10^{-1}\right)$, $D_{c}$ converges to an average integer value of $\overline{D_{c}} \approx 2$, confirming that the torus is indeed two-dimensional. This is consistent with our earlier observation of two incommensurate natural frequencies $\left(f_{1}\right.$ and $\left.f_{2}\right)$ in the PSD (figure $2 b$ ).

To complement the pressure data, we show in figure $2(e)$ a sequence of timeresolved Abel-inverted images of the flame captured via broadband chemiluminescence emission $(\$ 2)$. If the system were simply oscillating in a periodic limit cycle at a dominant natural frequency of $f_{1}=248 \pm 1.5 \mathrm{~Hz}$, then the image sequence, which is recorded at $3000 \mathrm{~Hz}$, would repeat itself around once every 12 frames, i.e. at $t_{1}$, $t_{13}, t_{25}$ and $t_{37}$. However, as figure $2(e)$ shows, the flame contours at these four time instants (highlighted in yellow) are markedly different from each other, in accordance with our assessment that the system is exhibiting behaviour more complex than just a periodic limit cycle.

In summary, by examining $p^{\prime}(t)$ in the time, frequency and phase domains as well as by inspecting time-resolved Abel-inverted flame images, we have established that the natural self-excited dynamics of the system is quasiperiodic, with its phase trajectory evolving on a stable ergodic $\mathbb{T}^{2}$ torus attractor formed by two incommensurate natural modes: $f_{1}$ and $f_{2}$. From here onwards, we will refer to this self-excited state as $\mathbb{T}_{1,2}^{2}$, with subscripts 1 and 2 denoting the dominant roles played by modes $f_{1}$ and $f_{2}$.

\subsection{Forced synchronization: period-1, $\mathbb{T}^{2}$ and $\mathbb{T}^{3}$ quasiperiodic states}

Having established that the self-excited dynamics is dominated by ergodic $\mathbb{T}^{2}$ quasiperiodicity, we proceed to examine the forced synchronization dynamics. We begin with two synchronization maps, both in a parameter space defined by the forcing frequency $\left(f_{f} / f_{1}\right.$ or $\left.f_{f} / f_{2}\right)$ and the forcing amplitude $\left(\epsilon_{f} \equiv\left|u^{\prime}\right| / \bar{u}\right)$, but with one (figure $3 a$ ) showing the forcing conditions at which different dynamical states arise and the other (figure $3 b$ ) showing contours of the normalized response amplitude. The normalized response amplitude is defined as $\eta_{p^{\prime}} \equiv\left(\sigma_{p^{\prime}}^{*}-\sigma_{p^{\prime}}\right) / \sigma_{p^{\prime}}$, where $\sigma_{p^{\prime}}^{*}$ and $\sigma_{p^{\prime}}$ denote the root mean square of $p^{\prime}(t)$ when the system is forced and unforced, respectively. Consequently, the thermoacoustic oscillations are weakened by the forcing when $\eta_{p^{\prime}}<0$ (blue regions) but are amplified by the forcing when $\eta_{p^{\prime}}>0$ (red regions). Overlaid on the contours of $\eta_{p^{\prime}}$ (figure $3 b$ ) are discrete markers showing the onset of complete synchronization for two different self-excited states: the $\mathbb{T}_{1,2}^{2}$ state of the present study (white-red markers) and the period-1 state $\left(P 1_{1}\right)$ studied by Guan et al. (2019a) (black markers). The latter is shown for comparison with the forced synchronization of a periodic limit cycle.

At each value of $f_{f} / f_{1}$, we incrementally increase $\epsilon_{f}$ until reaching flame blow-off (FBO), a limiting state represented in figure $3(a, b)$ by grey background shading. A preliminary inspection of figure $3(a)$ shows that there are four different dynamical states before FBO. Representative computations of their correlation dimensions are shown in figure $3(c-f)$. First we examine the typical path taken through these four states as $\epsilon_{f}$ increases at a fixed $f_{f} / f_{1}$ that leads to $\eta_{p^{\prime}}<0$, in accordance with our focus on open-loop control $(\S 1.4)$. Figure 4 shows the $(a)$ time trace, 

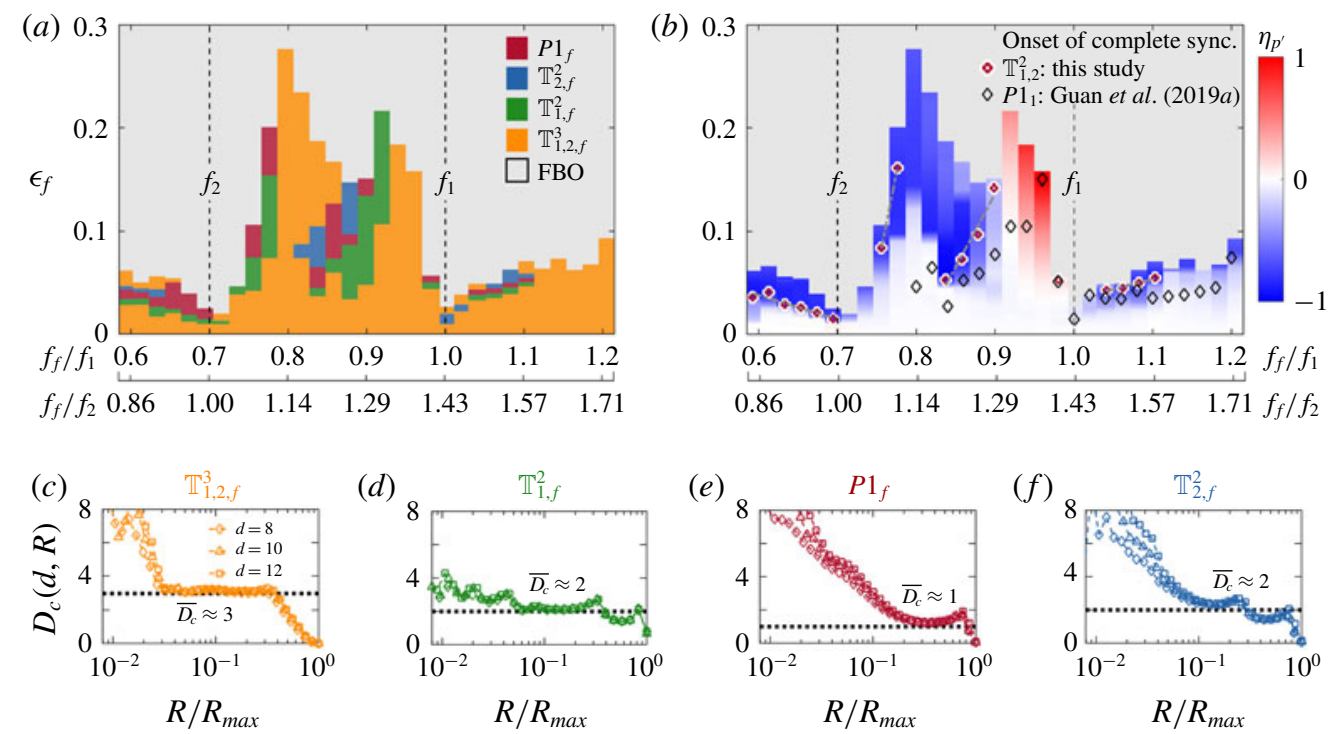

FIgURE 3. (Colour online) Forced response of an ergodic $\mathbb{T}_{1,2}^{2}$ thermoacoustic system: synchronization maps showing $(a)$ the forcing conditions at which four different dynamical states arise and $(b)$ contours of the normalized response amplitude $\left(\eta_{p^{\prime}}\right)$, both in a parameter space defined by the forcing frequency $\left(f_{f} / f_{1}\right.$ or $\left.f_{f} / f_{2}\right)$ and the forcing amplitude $\left(\epsilon_{f} \equiv\left|u^{\prime}\right| / \bar{u}\right)$. In $(b)$, the discrete markers indicate the onset of complete synchronization for two self-excited states: the $\mathbb{T}_{1,2}^{2}$ state of the present study (white-red markers) and the period-1 state $\left(P 1_{1}\right)$ studied by Guan et al. (2019a) (black markers). In $(a, b)$, the grey background regions denote flame blow-off (FBO). Also shown are representative computations of the correlation dimension for each of the four states in $(a)$, which correspond to the forcing conditions of figure $4:(c) \mathbb{T}_{1,2, f}^{3},(d) \mathbb{T}_{1, f}^{2},(e) P 1_{f}$, and $(f) \mathbb{T}_{2, f}^{2}$.

(b) PSD, (c) instantaneous phase difference between the forcing and the system, $\Delta \psi_{f, p^{\prime}} \equiv \psi_{f}-\psi_{p^{\prime}}$ (bottom row: $-\psi_{p^{\prime}}$ only), $(d)$ phase portrait and (e) Poincaré map of $p^{\prime}(t)$ at $f_{f} / f_{1}=0.63\left(f_{f} / f_{2}=0.90\right)$ for six values of $\epsilon_{f}$, including the unforced case $\left(\epsilon_{f}=0\right)$. From this and figure 3, we find much richer synchronization dynamics than that which has been reported in the thermoacoustics literature, which we will discuss below in order of increasing $\epsilon_{f}$.

\subsubsection{En route to partial synchronization: three-frequency quasiperiodicity $\left(\mathbb{T}_{1,2, f}^{3}\right)$}

When forced at a low amplitude (figure 4: $\epsilon_{f}=0.016$ ), the system transitions from its unforced two-frequency quasiperiodic state $\left(\mathbb{T}_{1,2}^{2}\right)$ with two natural modes $\left(f_{1}\right.$ and $\left.f_{2}\right)$ to a three-frequency quasiperiodic state $\left(\mathbb{T}_{1,2, f}^{3}\right)$ with the same two natural modes $\left(f_{1}\right.$ and $\left.f_{2}\right)$ and a forced mode $\left(f_{f}\right)$. This can be seen in the PSD (figure $4 b$ ), where a sharp peak at $f_{f}$ emerges alongside the two existing peaks at $f_{1}$ and $f_{2}$. Surrounding these three incommensurate peaks are a series of weaker peaks at their linear combinations $\left(\left|n f_{1} \pm m f_{2} \pm a f_{f}\right|\right.$, where $a$ is an integer), indicating the presence of nonlinear four-wave interactions between the two natural modes and the forced mode (Schmid \& Henningson 2012). These interactions persist down to low frequencies, appearing in the time trace as slow modulations of the pressure amplitude (figure $4 a$ ).

As expected for an asynchronous state, $\Delta \psi_{f, p^{\prime}}$ drifts unboundedly with time (figure $4 c$ ). This is known as phase drifting and can be found in all forced self-excited 
(a)

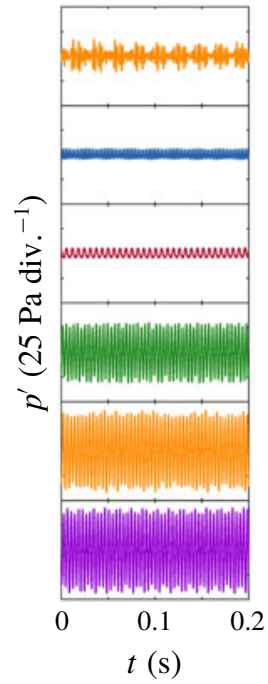

(b)

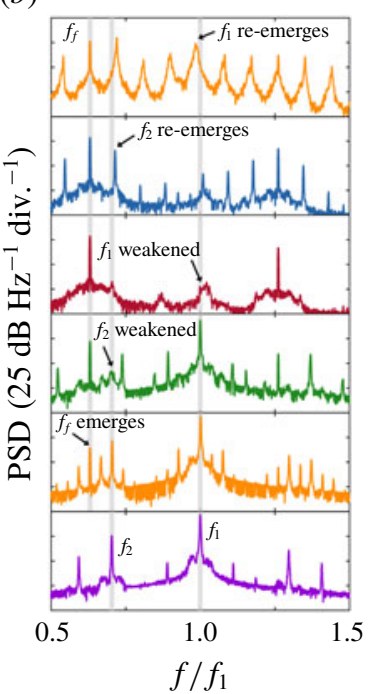

(c)

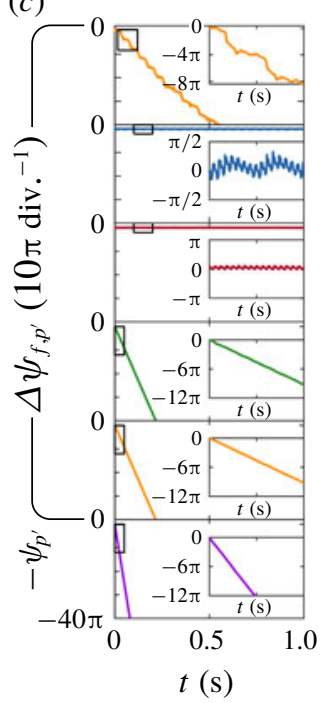

(d)

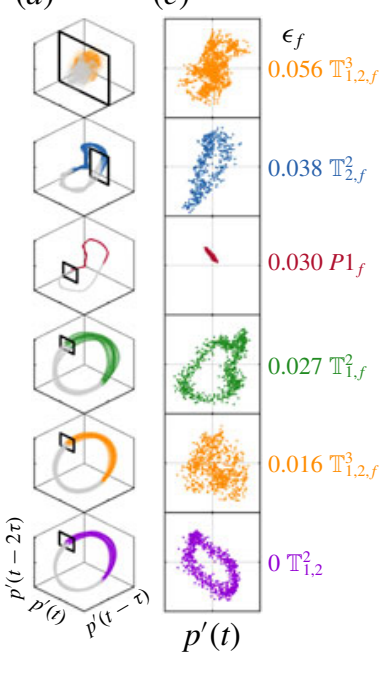

FIGURE 4. (Colour online) Forced response of an ergodic $\mathbb{T}_{1,2}^{2}$ thermoacoustic system undergoing asynchronous quenching at $f_{f} / f_{1}=0.63\left(f_{f} / f_{2}=0.90\right)$ for six values of $\epsilon_{f}$, including the unforced case $\left(\epsilon_{f}=0\right)$ : $(a)$ time trace, $(b) \operatorname{PSD},(c) \Delta \psi_{f, p^{\prime}}$ (bottom row: $\left.-\psi_{p^{\prime}}\right),(d)$ phase portrait and $(e)$ Poincaré map of $p^{\prime}(t)$ in the combustor. In $(d, e)$, phase-space reconstruction is performed with $d=3$ and $\tau=0.005 \mathrm{~s}$. The bottom row of $(c)$ shows $-\psi_{p^{\prime}}$, rather than $\Delta \psi_{f, p^{\prime}}$, as $\epsilon_{f}=0$ there.

oscillators prior to complete synchronization (Pikovsky et al. 2003). At this particular forcing amplitude $\left(\epsilon_{f}=0.016\right), \Delta \psi_{f, p^{\prime}}$ drifts almost linearly in time, with no sign of phase slipping. The absence of phase slipping, however, is not representative of all instances of $\mathbb{T}_{1,2, f}^{3}$. As $\S 3.2 .4$ will show, at higher values of $\epsilon_{f}, \Delta \psi_{f, p^{\prime}}$ can sometimes drift nonlinearly in time, with phase slips occurring in integer multiples of $\pm 2 \pi$.

In the phase portrait (figure $4 d$ ), the system trajectory evolves on a non-repeating orbit around a stable torus attractor. The corresponding Poincaré map shows a densely filled irregular structure (figure $4 e$ ), while the correlation dimension approaches an integer value of $\overline{D_{c}} \approx 3$ (figure $3 c$ ). Collectively, these observations indicate that the torus is ergodic and three-dimensional, with one active DOF arising from each of the three incommensurate modes: $f_{1}, f_{2}$ and $f_{f}$. This state is thus referred to as $\mathbb{T}_{1,2, f}^{3}$.

State $\mathbb{T}_{1,2, f}^{3}$ is similar to state (B) of Anishchenko et al. (2008) ( $\$ 1.3$ ), albeit with a subtle, but important, difference: $\mathbb{T}_{1,2, f}^{3}$ can be observed even for exceedingly weak forcing (figure $3 a$ : down to just above $\epsilon_{f}=0$ ), whereas state (B) of Anishchenko et al. (2008) requires moderate forcing owing to the need to destroy any mutual synchronization between the two natural modes and thus to enable their frequencies to become incommensurately different from each other as well as from $f_{f}$. In other words, if the self-excited state were a resonant limit cycle, as it was in the study by Anishchenko et al. (2008), then to observe three-frequency quasiperiodicity would require a forcing amplitude that is sufficient to desynchronize the two initially commensurate natural modes. In our experiments, however, the self-excited state is already ergodic, with an irrational winding number, so even exceedingly weak forcing can produce three-frequency quasiperiodicity. In $\S 4$, we will show that, along with 
other resonant and ergodic quasiperiodic states, $\mathbb{T}_{1,2, f}^{3}$ can be qualitatively reproduced with two coupled VDP oscillators forced by a sinusoidal term.

In nonlinear dynamical systems, three-frequency quasiperiodic states are known to be potentially unstable to arbitrary weak perturbations and can transition to strange attractors - in what is commonly referred to as the Ruelle-Takens-Newhouse route to chaos (Newhouse, Ruelle \& Takens 1978). In this study, we test for the presence of chaos using various methods, including the 0-1 test (Gottwald \& Melbourne 2004) and the permutation spectrum test (Kulp \& Zunino 2014), but find a negative result. This is not entirely surprising given that many experimental and numerical studies have unequivocally demonstrated the existence of stable quasiperiodic states with three incommensurate frequencies (Battelino 1988; Borkowski et al. 2015) and some with even four or five (Walden et al. 1984; Van Buskirk \& Jeffries 1985). Nevertheless, it is important to study such high-dimensional $(\geqslant 3)$ quasiperiodic states because the route to chaos (and hence to turbulence; Ruelle \& Takens 1971) may pass through them. For example, transitions to chaos via stable three-frequency quasiperiodicity have been observed in experiments on a variety of systems, ranging from barium-sodium niobate crystals (Martin, Leber \& Martienssen 1984) to Rayleigh-Bénard convection (Gollub \& Benson 1980; Libchaber \& Maurer 1982). Our measurements of $\mathbb{T}_{1,2, f}^{3}$ constitute the first experimental evidence of three-frequency quasiperiodicity in a forced self-excited $\mathbb{T}_{1,2}^{2}$ thermoacoustic system, potentially paving the way for the development of alternative strategies to control multi-mode combustor oscillations using concepts from chaos theory (Hovel 2010).

\subsubsection{Partial synchronization: two-frequency quasiperiodicity $\left(\mathbb{T}_{1, f}^{2}\right)$}

When forced at a moderate amplitude (figure 4: $\epsilon_{f}=0.027$ ), the system undergoes partial synchronization: the weaker of the two natural modes $\left(f_{2}\right)$ synchronizes with the forcing $\left(f_{f}\right)$, while the stronger natural mode $\left(f_{1}\right)$ remains desynchronized. In the PSD (figure $4 b$ ), this is evidenced by a weakening of the $f_{2}$ mode, which reduces the number of intermediate spectral peaks arising from nonlinear interactions between the forced and natural modes, as there is now one fewer natural mode (relative to $\mathbb{T}_{1,2, f}^{3}$ ) capable of producing such interactions. In phase space (figure $4 d$ ), this leads to the emergence of a resonant $\mathbb{T}_{1, f}^{2}$ torus on the existing $\mathbb{T}_{1,2, f}^{3}$ torus $(\$ 3.2 .1)$, as indicated by the presence of a closed continuous ring in the Poincaré map (figure $4 e$ ) and by a correlation dimension that approaches an integer value of $\overline{D_{c}} \approx 2$ within the self-similar Euclidean scaling range (figure $3 d$ ).

Like $\mathbb{T}_{1,2, f}^{3}, \mathbb{T}_{1, f}^{2}$ exhibits phase drifting: $\Delta \psi_{f, p^{\prime}}$ drifts unboundedly with time (figure $4 c$ ). When $\mathbb{T}_{1,2, f}^{3} \rightarrow \mathbb{T}_{1, f}^{2}$, the time-averaged slope of $\Delta \psi_{f, p^{\prime}},\left\langle\Delta \dot{\psi}_{f, p^{\prime}}\right\rangle$, remains largely unchanged, implying that the amplitude-weighted time average of all the spectral components in the signal also remains largely unchanged. At first sight, this finding might seem counterintuitive, given that the $f_{2}$ mode becomes synchronized as $\mathbb{T}_{1,2, f}^{3} \rightarrow \mathbb{T}_{1, f}^{2}$. However, this apparent anomaly can be explained by the fact that the $f_{2}$ mode is initially 10 times weaker than the $f_{1}$ mode $(\$ 3.1)$, so its synchronization has only a negligible influence on the overall system frequency, $\left\langle\Delta \dot{\psi}_{f, p^{\prime}}\right\rangle$. The relatively constant $\left\langle\Delta \dot{\psi}_{f, p^{\prime}}\right\rangle$ also suggests that the route to synchronization involves an inverse Neimark-Sacker bifurcation (Balanov et al. 2008). This is because, unlike a classical saddle-node bifurcation, an inverse Neimark-Sacker bifurcation causes a system to synchronize via asynchronous quenching of its natural modes, rather than by frequency pulling (Balanov et al. 2008). This is precisely the behaviour observed here, as will be elaborated on in $\$ \S 3.2 .3$ and 3.3. As for the universality of $\mathbb{T}_{1, f}^{2}$, we 
note that this partially synchronous state is equivalent to state (C) of Anishchenko et al. (2008) (\$1.3) and can thus be modelled with two coupled VDP oscillators forced by a sinusoidal term, as will be demonstrated in $\S 4$.

\subsubsection{Complete synchronization: a period-1 limit cycle $\left(P 1_{f}\right)$}

When forced at a critically high amplitude (figure 4: $\epsilon_{f}=0.030$ ), the system undergoes complete synchronization: the $f_{1}$ mode now joins the $f_{2}$ mode in being synchronized with the forcing at $f_{f}$. This can be seen in the PSD (figure $4 b$ ), where sharp peaks at $f_{f}$ and its harmonics dominate over residual traces of $f_{1}$ and $f_{2}$. The corresponding phase trajectory evolves on a closed orbit that repeats itself once every cycle (figure $4 d$ ), which is characteristic of a period-1 limit cycle. The period-1 nature of this orbit is confirmed by the presence of a single cluster of intercepts in the Poincaré map (figure 4e). This cluster would be a perfectly discrete point if the oscillations in $p^{\prime}(t)$ were free of cyclic variability, which, in this system, arises from background noise and residual traces of the $f_{1}$ and $f_{2}$ modes. The limit-cycle nature of this attractor is corroborated by a correlation dimension that approaches an integer value of $\overline{D_{c}} \approx 1$ (figure $3 e$ ), indicating a single active DOF in the system. From these indicators, it can be concluded that this completely synchronous state is a period-1 limit cycle at $f_{f}$. This state is therefore referred to as $P 1_{f}$.

Crucially, the transition from partial synchronization $\left(\mathbb{T}_{1, f}^{2}\right)$ to complete synchronization $\left(P 1_{f}\right)$ is accompanied by a substantial reduction in the thermoacoustic amplitude, which is evident in the time trace (figure $4 a$ ) and in the contours of $\eta_{p^{\prime}}$ (figure $3 b$ : blue regions). This reduction arises from asynchronous quenching and will be analysed in $\$ 3.3$ via the spectral powers and Rayleigh indices of the $f_{1}, f_{2}$ and $f_{f}$ modes.

Unlike $\mathbb{T}_{1,2, f}^{3}$ and $\mathbb{T}_{1, f}^{2}, \quad P 1_{f}$ exhibits phase locking instead of phase drifting (figure $4 c$ ): $\Delta \psi_{f, p^{\prime}}$ becomes constant in time, indicating that the system is oscillating primarily at $f_{f}$ and its harmonics. There are, however, minor fluctuations in $\Delta \psi_{f, p^{\prime}}$ (see the insets of figure $4 c$ ) occurring on the same (fast) time scale as $p^{\prime}(t)$ itself. These fluctuations occur because the system is not oscillating as sinusoidally as the forcing signal, as is evident from the skewed waveform of the time trace (figure $4 a$ ) and the presence of harmonics of $f_{f}$ in the PSD (figure $4 b$ ). Phase locking is a signature feature of complete synchronization and, like phase drifting, can be modelled with canonical oscillators (Pikovsky et al. 2003).

State $P 1_{f}$ is similar to the classical $1: 1$ synchronous states found in other forced self-excited flow systems, such as cylinder wakes (Provansal, Mathis \& Boyer 1987), reacting wakes (Pawar et al. 2018), cross-flow jets (Davitian et al. 2010), jet diffusion flames (Li \& Juniper 2013b) and premixed combustors (Bellows et al. 2008). However, unlike a classical synchronous state, $P 1_{f}$ here is arrived at via synchronization of not just one natural mode, but two natural modes with incommensurate frequencies. Furthermore, these two natural modes are not synchronized simultaneously but in succession, with the weaker mode $\left(f_{2}\right)$ synchronizing before - i.e. at lower $\epsilon_{f}$ than - the stronger mode $\left(f_{1}\right)$ under most forcing conditions, including some where $f_{f}$ is closer to $f_{1}$ than it is to $f_{2}$ (figure $3 a$ ). Like $\mathbb{T}_{1,2, f}^{3}$ and $\mathbb{T}_{1, f}^{2}$, $P 1_{f}$ is well predicted by the analysis of Anishchenko et al. (2008) ( $\$ 1.3$ : state (D)). Moreover, as $\S 4$ will show, the sequential synchronization of $f_{2}$ and then $f_{1}$ can be qualitatively modelled with canonical oscillators, implying that these synchronization dynamics are not limited to this particular system, but are universal features of a wide range of forced and coupled self-excited systems with multiple natural modes (Pikovsky et al. 2003; Loose et al. 2010). 
Figure 3(a) shows that the minimum $\epsilon_{f}$ required for complete synchronization $\left(P 1_{f}\right)$ decreases as $f_{f}$ approaches either $f_{1}$ or $f_{2}$, resulting in two $\vee$-shaped Arnold tongues, one centred on each of the two natural modes: $f_{f} / f_{1}=1$ and $f_{f} / f_{2}=1$ (figure $3 b$ : white-red markers). In addition, there is a (third) partial Arnold tongue centred on $f_{f} / f_{1} \approx 0.80\left(f_{f} / f_{2} \approx 1.14\right)$, which sits between the two primary tongues and could be caused by high-order resonances between them (Pikovsky et al. 2003). The presence of Arnold tongues is characteristic of forced self-excited periodic oscillators (Balanov et al. 2008) and has been observed in various such systems (Pikovsky et al. 2003), including those from hydrodynamics (Li \& Juniper 2013a) and thermoacoustics (Guan et al. 2019a). However, this is the first time that two primary Arnold tongues, centred on two incommensurate natural modes, have been observed together in a thermoacoustic system. The coexistence of two Arnold tongues enhances the versatility of open-loop control by enabling complete synchronization to be reached at lower $\epsilon_{f}$ and across a wider range of $f_{f}$.

For comparison with a period-1 thermoacoustic system, we show in figure $3(b)$ the synchronization boundaries (black markers) from the experiments of Guan et al. $(2019 a)$. It can be seen that introducing a second natural mode $\left(f_{2}\right)$ to a self-excited system with an existing natural mode $\left(f_{1}\right)$ alters the synchronization boundaries in two main ways: (i) it increases the minimum $\epsilon_{f}$ required for complete synchronization, with the secondary effect of making $P 1_{f}$ unreachable for some forcing frequencies (mostly those very close to $f_{f} / f_{1}=1$ ) because FBO occurs first; and (ii) it makes complete synchronization around the second natural mode $\left(f_{f} / f_{2}=1\right)$ possible, although, as was the case for the first natural mode, $P 1_{f}$ becomes unreachable very close to $f_{f} / f_{2}=1$ because FBO occurs first. A possible explanation for the increase in the minimum $\epsilon_{f}$ required for complete synchronization can be found in a recent study on the flame double-input describing function. Using numerical simulations of a laminar conical flame based on the $G$-equation, Orchini \& Juniper (2016) showed that the presence of an acoustic mode at a second frequency can affect the gain of the flame at the first frequency. This effect often reduces the gain of the flame to both acoustic modes as compared to its gain if the two modes were present one at a time. Similar reductions in flame gain, arising from two-frequency forcing, have been observed in the experiments of Moeck \& Paschereit (2012). If the flame gain is reduced more at off-resonance frequencies, then that could explain why a higher $\epsilon_{f}$ is required to cause complete synchronization in the $\mathbb{T}_{1,2}^{2}$ quasiperiodic system studied here as compared to the period-1 system studied by Guan et al. (2019a).

Figure $3(b)$ also shows that, across a wide range of $f_{f}$ around both $f_{1}$ and $f_{2}$, the thermoacoustic amplitude can be drastically reduced - typically to less than $15 \%\left(\eta_{p^{\prime}}<-0.85\right)$ of that of the unforced system and sometimes to as low as $2 \%$ $\left(\eta_{p^{\prime}}=-0.98\right.$ at $\left.f_{f} / f_{1}=0.84\right)$. In general, for a fixed $f_{f}$, the lowest $\eta_{p^{\prime}}$ values coincide with complete synchronization $\left(P 1_{f}\right)$ and occur via asynchronous quenching of the natural modes $(\$ 3.3)$. There is, however, a limited range of $f_{f}$ close to $f_{1}$ (figure $3 b$, red regions: $\left.0.91 \leqslant f_{f} / f_{1} \leqslant 0.99\right)$ in which the thermoacoustic amplitude increases above that of the unforced system. This increase in $\eta_{p^{\prime}}$ coincides with $\mathbb{T}_{1,2, f}^{3}$ and $\mathbb{T}_{1, f}^{2}$ and occurs via resonant amplification of the forcing signal ( $\left.\$ 3.3\right)$. In summary, whether external forcing increases or decreases the thermoacoustic amplitude depends on $f_{f}$ relative to $f_{1}$ and $f_{2}$, but the degree of the increase or decrease depends on $\epsilon_{f}$, with the maximum decrease occurring at complete synchronization $\left(P 1_{f}\right)$. In $\S 3.3$, we will examine in more detail specific examples of both asynchronous quenching and resonant amplification, with the aim of better understanding the physical mechanisms behind the observed variations in $\eta_{p^{\prime}}$. 


\subsubsection{Beyond complete synchronization: $\mathbb{T}_{2, f}^{2}, \mathbb{T}_{1,2, f}^{3}$ and flame blow-off}

When forced at an amplitude above that required for complete synchronization (figure 4: $\epsilon_{f} \geqslant 0.038$ ), the system transitions out of $P 1_{f}$ and into one of three states.

(i) Partial synchronization (figure 4: $\epsilon_{f}=0.038$ ): high-amplitude forcing brings the $f_{2}$ mode out of synchronization but leaves the $f_{1}$ mode in synchronization with the $f_{f}$ mode, resulting in a new two-frequency quasiperiodic state dominated by $f_{2}$ and $f_{f}$ : $\mathbb{T}_{2, f}^{2}$. This two-frequency quasiperiodic state differs from the one observed en route to $P 1_{f}$ ( $\$ 3.2 .2: \mathbb{T}_{1, f}^{2}$ ) in that here it is the $f_{2}$ mode, rather than the $f_{1}$ mode, that interacts with the $f_{f}$ mode to produce resonant quasiperiodicity. This is evidenced by the re-emergence of the peak at $f_{2}$ in the PSD (figure $4 b$ ), alongside the existing peaks at $f_{f}$ and its harmonics. The two-dimensionality of this state is confirmed by the presence of a closed continuous ring in the Poincare map (figure 4e) and by a correlation dimension that approaches an integer value of $\overline{D_{c}} \approx 2$ (figure $3 f$ ), indicating two active DOFs in the system.

The partial synchronicity of $\mathbb{T}_{2, f}^{2}$ is also corroborated by the fact that $\Delta \psi_{f, p^{\prime}}$ oscillates on two distinct time scales (figure 4c): $(a)$ a fast time scale arising from the non-sinusoidal waveform of $p^{\prime}(t)$ relative to the sinusoidal forcing signal, as discussed in $\$ 3.2 .3$; and $(b)$ a slow time scale associated with low-frequency beating. Despite these oscillations, however, $\Delta \psi_{f, p^{\prime}}$ remains bounded in time. Thus, although $\Delta \psi_{f, p^{\prime}}$ itself does not remain perfectly constant in time, its time-averaged slope $\left\langle\Delta \dot{\psi}_{f, p^{\prime}}\right\rangle$ is still zero, indicating phase trapping - a partially synchronous feature defined by frequency locking without phase locking (Thévenin et al. 2011; Li \& Juniper 2013c). This feature can be found not only at $\mathbb{T}_{2, f}^{2}$ but also at $\mathbb{T}_{1, f}^{2}$, as $\S 4$ will show. Phase trapping has been observed in various periodic thermoacoustic systems, such as a ducted laminar premixed flame (Kashinath et al. 2018) and a turbulent premixed combustor (Balusamy et al. 2015). Here we show that phase trapping is not limited to periodic systems, but can arise in quasiperiodic systems as well, broadening the universality of this synchronization feature.

When $\epsilon_{f}$ increases for a fixed $f_{f}, P 1_{f}$ is usually preceded by $\mathbb{T}_{1, f}^{2}$ but is succeeded by $\mathbb{T}_{2, f}^{2}$ (figure $3 a$ ). In other words, the mode that synchronizes first $\left(f_{2}\right)$ desynchronizes first, and the mode that synchronizes last $\left(f_{1}\right)$ desynchronizes last. This implies that mode switching occurs as the system passes through $P 1_{f}$. A possible cause of this is that the gains of the two modes $\left(f_{1}\right.$ and $\left.f_{2}\right)$ vary non-monotonically as $\epsilon_{f}$ increases, behaving as if they were nonlinearly unstable modes (Lieuwen \& Yang 2005). An analogous phenomenon, in which the natural mode of a synchronized periodic oscillator re-emerges after $\epsilon_{f}$ increases above the value required for synchronization, has recently been observed in an identical thermoacoustic system (Guan et al. 2019b). In both the periodic case (Guan et al. 2019b) and the quasiperiodic case (figure 3a), the minimum $\epsilon_{f}$ required to re-excite the natural mode(s) increases as $f_{f}$ deviates from either $f_{1}$ or $f_{2}$. This behaviour is similar to that seen in the boundaries of the Arnold tongues ( $\$ 3.2 .3$ ).

(ii) Three-frequency quasiperiodicity (figure $4: \epsilon_{f}=0.056$ ): high-amplitude forcing re-excites both the $f_{1}$ and $f_{2}$ modes, causing them to interact with each other and with the $f_{f}$ mode to produce a three-frequency quasiperiodic state: $\mathbb{T}_{1,2, f}^{3}$. This is similar to the $\mathbb{T}_{1,2, f}^{3}$ state observed en route to partial synchronization (§3.2.1), but with spectral peaks that are more broadband (figure $4 b$ ) and with an attractor structure that is more fractal-like, reminiscent of a strange attractor (figure $4 d, e$ ). These differences suggest that the system could be approaching the edge of chaos via the Ruelle-Takens-Newhouse route (Newhouse et al. 1978; Hilborn 2000), although 
results from the 0-1 test (Gottwald \& Melbourne 2004) and the permutation spectrum test (Kulp \& Zunino 2014) show no definitive evidence of chaos yet. It is worth noting that this $\mathbb{T}_{1,2, f}^{3}$ state can be reached via $\mathbb{T}_{2, f}^{2}$ (see above) or directly from $P 1_{f}$. In the latter case, we suspect that there may in fact be an intermediate regime of $\mathbb{T}_{2, f}^{2}$ between $P 1_{f}$ and $\mathbb{T}_{1,2, f}^{3}$, but that this regime is so much smaller than the average increment of the forcing amplitude $\left(\epsilon_{f}=0.005\right)$ that it could not be detected with statistical confidence in our experiments.

A notable feature of this particular $\mathbb{T}_{1,2, f}^{3}$ case is its $\Delta \psi_{f, p^{\prime}}$ dynamics (figure $4 c$ ). Like the $\mathbb{T}_{1,2, f}^{3}$ case from $\S 3.2 .1$, here $\Delta \psi_{f, p^{\prime}}$ drifts unboundedly with time. However, unlike before, this drifting is not perfectly linear in time: there is a gradual drift followed by an abrupt drop, resulting in $\Delta \psi_{f, p^{\prime}}$ slipping by two full cycles $(4 \pi)$ at a time. In synchronization theory, this behaviour is known as phase slipping (Pikovsky et al. 2003). Physically, it implies that the system follows the forcing signal fairly closely most of the time, but then suddenly speeds up and gains an integer number of cycles within a relatively short time (if $f_{f} / f_{1}>1$, it slows down and loses cycles). Phase slipping is thought to be a universal feature of forced and coupled self-excited oscillators (Pikovsky et al. 2003). In fluid mechanics, for example, it has been observed in various systems, including an elastically mounted cylinder interacting with its self-excited wake (Khalak \& Williamson 1999), a forced self-excited jet of helium (Li \& Juniper 2013c) and a forced combustor with self-excited periodic oscillations (Balusamy et al. 2015). The fact that we also observe phase slipping here in a forced thermoacoustic system with self-excited $\mathbb{T}_{1,2}^{2}$ oscillations - further supports the universality of this synchronization feature. The existence of such subtle $\Delta \psi_{f, p^{\prime}}$ dynamics could have important implications for the design of open-loop control strategies, because the stability of thermoacoustic systems is known to be exceedingly sensitive to the phase relationship between the flame and its surrounding acoustic modes (Juniper \& Sujith 2018).

(iii) Flame blow-off: at a sufficiently high $\epsilon_{f}$, the flame is blown off the burner lip by incident acoustic velocity perturbations, causing the thermoacoustic oscillations to die away (figure $3 a, b$ : grey background regions). Like the boundaries for partial synchronization $\left(\mathbb{T}_{1, f}^{2}\right)$ and complete synchronization $\left(P 1_{f}\right)$, the minimum $\epsilon_{f}$ required for FBO decreases as $f_{f}$ approaches either $f_{1}$ or $f_{2}$, which suggests that the resonant feedback provided by the combustor acoustics is strongest at the two natural modes. The most common state bordering FBO is $\mathbb{T}_{1,2, f}^{3}$ (figure $3 a$ ), which is consistent with the view that this state is the most likely to transition to chaos (via the Ruelle-TakensNewhouse route; Newhouse et al. 1978) and is therefore the most likely to cause irregular flame movements, leading to FBO. This interpretation is supported by timeresolved chemiluminescence images showing that the flame moves around chaotically just moments before FBO (see appendix A). This finding is consistent with previous observations of FBO arising from chaotic flame motion in both forced and unforced laminar premixed systems (Bourehla \& Baillot 1998; Kabiraj \& Sujith 2012).

\subsubsection{Optimal control strategy}

From figure $3(a, b)$, we can see that the synchronization dynamics observed in figure 4 are not limited to when $f_{f}$ is below $f_{1}$ and $f_{2}$, but also arise when $f_{f}$ is above or between $f_{1}$ and $f_{2}$. To illustrate this, we show in figures 5 and 6 data analogous to figure 4 but at $f_{f} / f_{1}=0.84$ and 1.10 , respectively, instead of $f_{f} / f_{1}=0.63$. It is clear that the system follows the same route to and out of complete synchronization $\left(P 1_{f}\right)$ as the previous case: unforced ergodic $\mathbb{T}_{1,2}^{2}$ quasiperiodicity $\rightarrow$ ergodic $\mathbb{T}_{1,2, f}^{3}$ quasiperiodicity $\rightarrow$ resonant $\mathbb{T}_{1, f}^{2}$ quasiperiodicity $\rightarrow$ synchronous $P 1_{f}$ periodicity $\rightarrow$ resonant $\mathbb{T}_{2, f}^{2}$ 
(a)

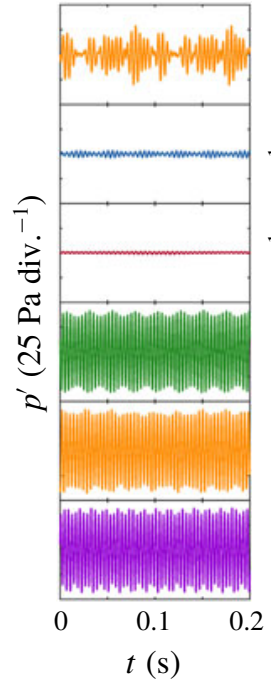

(b)

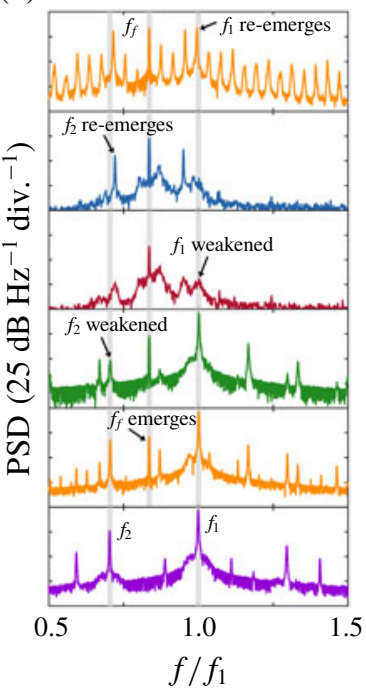

(c)

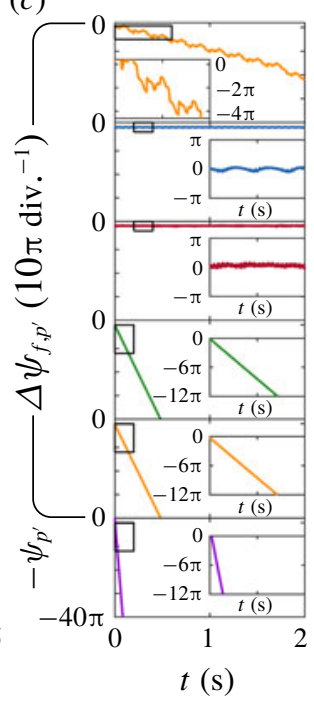

(d)

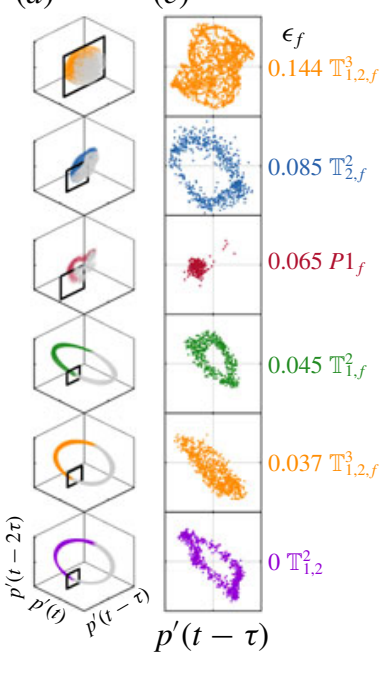

FIgURE 5. (Colour online) The same as for figure 4 but at $f_{f} / f_{1}=0.84\left(f_{f} / f_{2}=1.20\right)$.

(a)

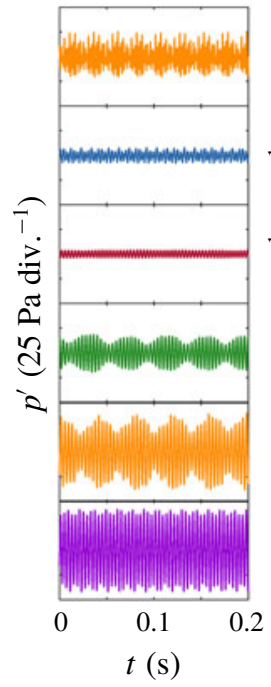

(b)

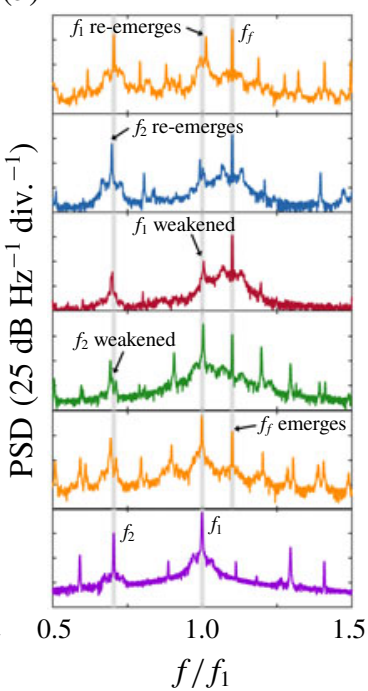

(c)

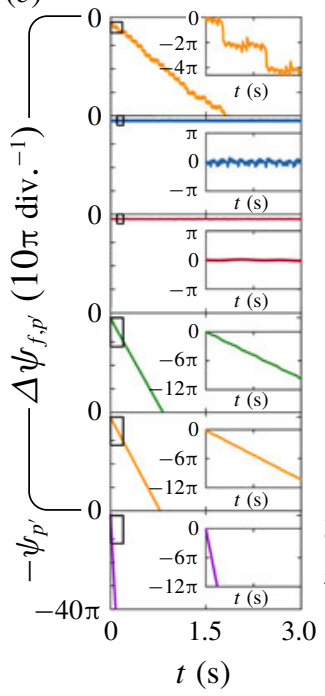

(d)

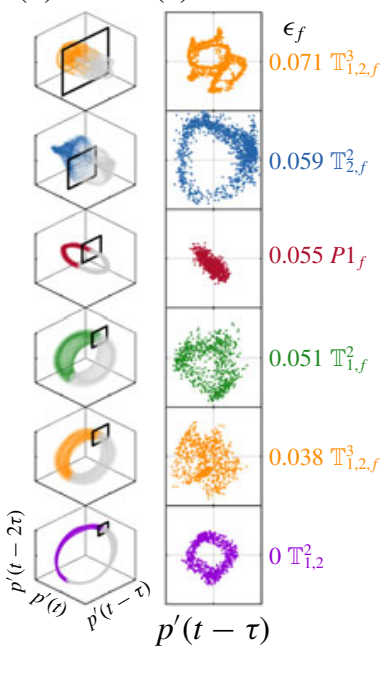

Figure 6. (Colour online) The same as for figure 4 but at $f_{f} / f_{1}=1.10\left(f_{f} / f_{2}=1.57\right)$.

quasiperiodicity $\rightarrow$ ergodic $\mathbb{T}_{1,2, f}^{3}$ quasiperiodicity $\rightarrow$ FBO. Furthermore, $\eta_{p^{\prime}}$ is similarly reduced by asynchronous quenching, with its maximum reduction occurring at $P 1_{f}$ (figure $3 b$ ). However, as noted in $\$ 3.2 .3$, not all values of $f_{f}$ can lead to $P 1_{f}$ or even $\mathbb{T}_{1, f}^{2}$, especially when $f_{2} \leqslant f_{f} \leqslant f_{1}$. The optimal control strategy for reducing $\eta_{p^{\prime}}$ is to force the system at an off-resonance frequency around its weaker natural mode $\left(f_{2}\right)$, at an amplitude just sufficient to cause $P 1_{f}$, because this produces the largest reduction in $\eta_{p^{\prime}}$ using relatively little actuation effort by exploiting asynchronous quenching (figure $3 b$ ). Across the entire $f_{f}$ range of this study, the actuator power required to cause asynchronous quenching at $P 1_{f}$ is generally negligible $(<0.05 \%)$ 
relative to the thermal power of the flame. If one is compelled to force at a frequency around the stronger natural mode $\left(f_{1}\right)$, then care must be taken to avoid a narrow range near $f_{1}\left(0.91 \leqslant f_{f} / f_{1} \leqslant 0.99\right)$ because here $\eta_{p^{\prime}}$ increases by resonant amplification. It is worth noting that a similar increase in $\eta_{p^{\prime}}$ can be found near $f_{1}$ of the equivalent periodic system (Guan et al. 2019a). This shows that the addition of a weak natural mode $\left(f_{2}\right)$ to the original single-mode $\left(f_{1}\right)$ system can make open-loop control easier to implement, because the forcing can now be applied around $f_{2}$, instead of $f_{1}$, with similar reductions in $\eta_{p^{\prime}}$ but less risk of amplification. Next we will examine in more detail both asynchronous quenching and resonant amplification.

\subsection{Asynchronous quenching and resonant amplification}

Figure 7 shows the spectral power contained in the $f_{1}$ mode $\left(P_{1}^{*}\right)$, the $f_{2}$ mode $\left(P_{2}^{*}\right)$, the $f_{f}$ mode $\left(P_{f}^{*}\right)$ and the total $p^{\prime}(t)$ signal $\left(P_{t}^{*}\right)$ for two distinct trends in $\eta_{p^{\prime}}$ : asynchronous quenching (figure $7 a, c$ ) and resonant amplification (figure $7 b, d$ ). These two trends are shown here for only two values of $f_{f}$ but are qualitatively representative of a range of $f_{f}$ around both $f_{1}$ and $f_{2}$. All four power indicators are normalized by the total power of the unforced system $\left(P_{t}\right.$, without an asterisk) and are shown as a function of the forcing power, which is normalized either by the forcing power required to cause the onset of complete synchronization, $\left(\epsilon_{f} / \epsilon_{f, C S}\right)^{2}$ (figure $7 a, c$ ), or by the forcing power required to cause FBO, $\left(\epsilon_{f} / \epsilon_{f, F B O}\right)^{2}$, if FBO occurs before complete synchronization (figure $7 b, d$ ). The modal power is found by integrating the PSD around each mode $( \pm 5 \mathrm{~Hz}$ ), which is equivalent to bandpass filtering around each frequency. The total power is found by integrating the PSD across its full bandwidth, and the result is checked to be equal (within $0.3 \%$ ) to the mean-squared fluctuation, in accordance with Parseval's theorem.

To investigate the cause of the $\eta_{p^{\prime}}$ variations, we show in figure $7(c, d)$ the Rayleigh index: $R I \equiv 1 / T \int_{0}^{T} p^{\prime}(t) q^{\prime}(t) \mathrm{d} t$, where $T$ is the sampling duration (Lieuwen \& Yang 2005). This is used to quantify the magnitude and direction of the energy exchange between the flame and the acoustic modes, accounting not only for the amplitude variations in $p^{\prime}(t)$ and $q^{\prime}(t)$ but also for their phase relationship (Hong et al. 2013). The Rayleigh indices of the three main modes $\left(R I_{1}^{*}, R I_{2}^{*}, R I_{f}^{*}\right)$ are computed by bandpass filtering $( \pm 5 \mathrm{~Hz})$ the $p^{\prime}(t)$ and $q^{\prime}(t)$ signals with a zero phase-distortion algorithm based on a forward-backward scheme (Gustafsson 1996). The Rayleigh index of the total signal $\left(R I_{t}^{*}\right)$ is computed without any filtering. All four Rayleigh indices are normalized by the total Rayleigh index of the unforced system $\left(R I_{t}\right.$, without an asterisk) and are shown as a function of the forcing power, which is normalized similarly to figure $7(a, b)$.

First we explore asynchronous quenching. When $\left(\epsilon_{f} / \epsilon_{f, C S}\right)^{2}$ increases within $\mathbb{T}_{1,2, f}^{3}$ (figure 7a: left yellow region), both $P_{1}^{*}$ and $P_{2}^{*}$ decrease slightly, but $P_{2}^{*}$ becomes suppressed first (see inset) as it is initially much smaller than $P_{1}^{*}$. This concurs with $R I_{2}^{*}$ (figure $7 c$ : inset), which decreases to zero concurrently with $P_{2}^{*}$ (figure $7 a$ : inset), indicating that the flame is feeding progressively less power into the $f_{2}$ mode as $\left(\epsilon_{f} / \epsilon_{f, C S}\right)^{2}$ increases. Thus, $P_{t}^{*}$ consists mostly of $P_{1}^{*}$, with only a minor contribution from $P_{f}^{*}$.

When $\left(\epsilon_{f} / \epsilon_{f, C S}\right)^{2}$ increases within $\mathbb{T}_{1, f}^{2}$ (figure $7 a$ : green region), $P_{1}^{*}$ decreases increasingly rapidly until it is eventually suppressed $\left(P_{1}^{*} \approx 0\right)$, marking the onset of complete synchronization. This suppression of $P_{1}^{*}$ causes a concurrent decrease in $P_{t}^{*}$ because $P_{2}^{*} \approx 0$ and $P_{f}^{*}$ increases only weakly with $\left(\epsilon_{f} / \epsilon_{f, C S}\right)^{2}$. The fact that $P_{t}^{*}$ decreases via a suppression of both $P_{2}^{*}$ and $P_{1}^{*}$, without resonant amplification of $P_{f}^{*}$, 

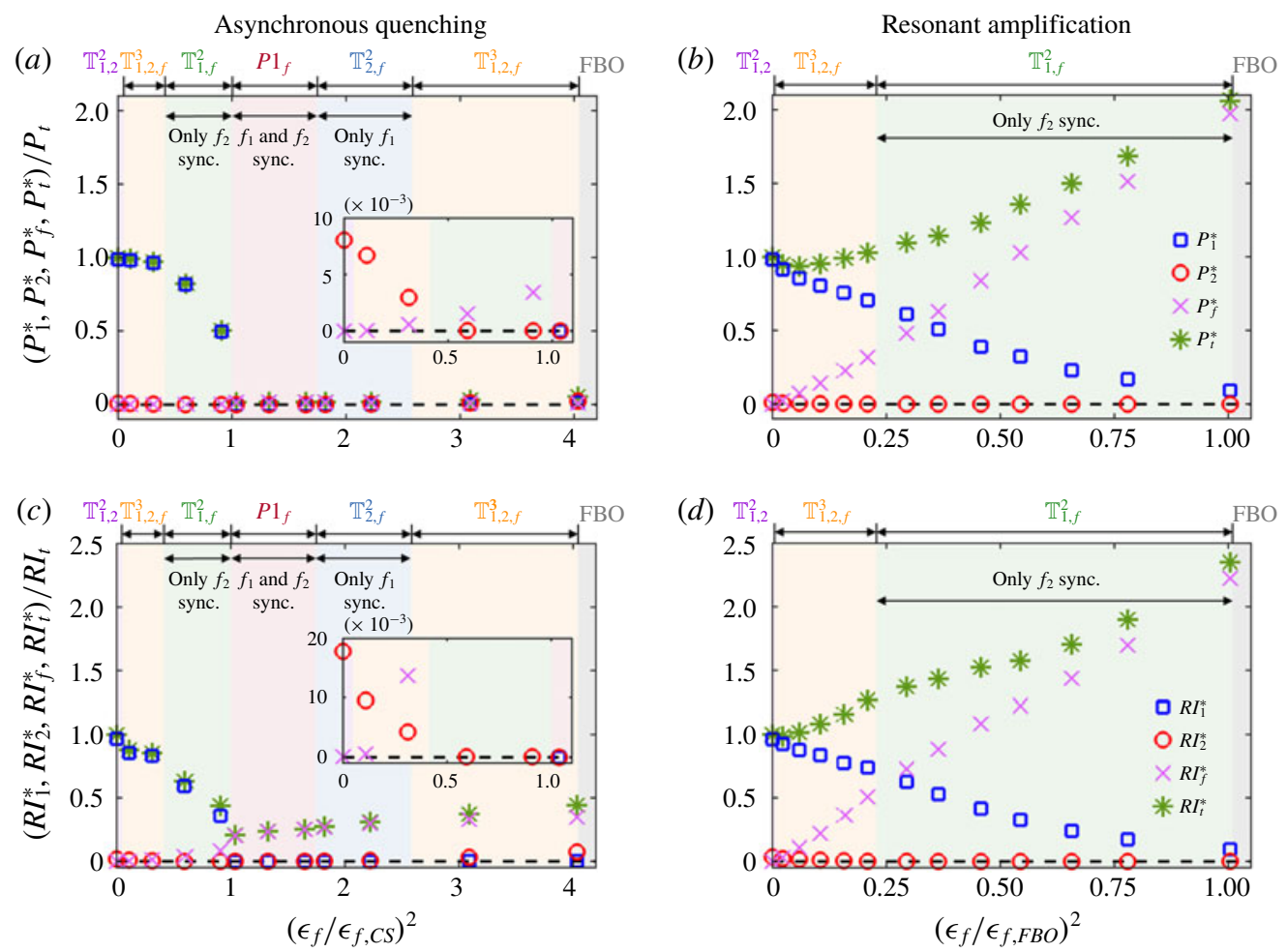

FIGURE 7. (Colour online) Representative cases of $(a, c)$ asynchronous quenching and $(b, d)$ resonant amplification in a forced self-excited ergodic $\mathbb{T}_{1,2}^{2}$ thermoacoustic system. Shown are $(a, b)$ the spectral power of the $p^{\prime}(t)$ signal and $(c, d)$ the Rayleigh index, both as a function of the forcing power. In $(a, c), f_{f} / f_{1}=0.63\left(f_{f} / f_{2}=0.90\right)$ and the forcing power is normalized by that required to cause the onset of complete synchronization. In $(b, d)$, $f_{f} / f_{1}=0.92\left(f_{f} / f_{2}=1.31\right)$ and the forcing power is normalized by that required to cause FBO.

is a hallmark of asynchronous quenching (Minorsky 1967). The transient response of the system as it transitions from $\mathbb{T}_{1,2}^{2}$ to $P 1_{f}$ via asynchronous quenching is discussed in appendix B. According to Keen \& Fletcher (1970), asynchronous quenching can be interpreted as an asymptotic loss of stability of the natural self-excited oscillations, arising from disturbances imposed by the external forcing, resulting in a decrease in the total oscillation amplitude of the forced self-excited system. For periodic thermoacoustic oscillations, asynchronous quenching has recently been shown to be caused by a reduced Rayleigh index (Guan et al. 2019a; Mondal et al. 2019). For quasiperiodic thermoacoustic oscillations, we find a similar reduction in the Rayleigh index (figure $7 c$ : green region), as evidenced by the steep decreases in both $R I_{1}^{*}$ and $R I_{t}^{*}$ en route to $P 1_{f}$. This shows that the physical cause of asynchronous quenching is a disruption of the positive coupling between the HRR from the flame and the $f_{1}$ acoustic mode of the combustor - and to a lesser extent the $f_{2}$ acoustic mode as well. Crucially, this generalizes the findings of Guan et al. (2019a) and Mondal et al. (2019) to $\mathbb{T}_{1,2}^{2}$ quasiperiodic thermoacoustic oscillations.

Asynchronous quenching, however, is not the only type of response possible: resonant amplification can occur as well, leading to an increase in $\eta_{p^{\prime}}$ (Odajima, 
Nishida \& Hatta 1974). This was mentioned briefly in our discussion of figure $3(b)$, where a region of $\eta_{p^{\prime}}>0$ appears near $f_{f} / f_{1}=1$. Within this region, the synchronization dynamics is topologically simpler than that observed during asynchronous quenching (figures 4 and 6). As $\epsilon_{f}$ increases from zero, the system transitions from $\mathbb{T}_{1,2}^{2}$ to $\mathbb{T}_{1,2, f}^{3}$. For some values of $f_{f}$, this is followed by a transition to $\mathbb{T}_{1, f}^{2}$ owing to the partial synchronization of the $f_{2}$ mode. However, complete synchronization does not necessarily follow, with the system remaining either desynchronized $\left(\mathbb{T}_{1,2, f}^{3}\right)$ or partially synchronized $\left(\mathbb{T}_{1, f}^{2}\right)$ until FBO. Crucially, rather than decreasing with $\epsilon_{f}$, the thermoacoustic amplitude increases (figure $3 b$ : red regions), peaking at a value nearly double that of the unforced system $\left(\eta_{p^{\prime}} \approx+1\right)$.

To better understand this increase in $\eta_{p^{\prime}}$, we examine the spectral power and the Rayleigh index (figure $7 b, d$ ). As $\left(\epsilon_{f} / \epsilon_{f, F B O}\right)^{2}$ increases, both natural modes undergo synchronous quenching, as indicated by the gradual decreases in $P_{1}^{*}$ and $P_{2}^{*}$. As before, this is accompanied by concurrent decreases in $R I_{1}^{*}$ and $R I_{2}^{*}$, indicating again that the natural modes are quenched through a reduction of the power supplied to them from the flame. However, the rate at which $P_{1}^{*}$ and $P_{2}^{*}$ decrease with $\left(\epsilon_{f} / \epsilon_{f, F B O}\right)^{2}$ is not sufficient to overcome the resonant amplification of $P_{f}^{*}$, causing $P_{t}^{*}$ to increase above that of the unforced system. Physically, this amplification of $P_{f}^{*}$ arises from increasingly strong coupling between the flame and the $f_{f}$ acoustic mode, as evidenced by the steep monotonic increase in $R I_{f}^{*}$ (figure $7 d$ ). An increase in $P_{t}^{*}$ due to amplification of $P_{f}^{*}$ is a defining feature of resonant amplification (Odajima et al. 1974) and can be modelled with a forced VDP oscillator (Keen \& Fletcher 1970). In thermoacoustics, such resonant amplification was recently observed by Guan et al. (2019a) and Mondal et al. (2019) in periodic systems. Here we show that the same amplification can occur in quasiperiodic systems as well, highlighting the need to choose $f_{f}$ carefully when applying open-loop control, regardless of the number of natural modes present.

\section{Low-order modelling}

It is well known that the nonlinear dynamics of self-excited systems can be modelled with low-order universal oscillators containing a VDP kernel (Pikovsky et al. 2003; Balanov et al. 2008). In thermoacoustics, this approach was first proposed by Culick (1971) as an analytical means of studying the nonlinear growth and saturation of unstable combustor modes. Since then, it has been used for various other applications, including output-only system identification (Noiray \& Schuermans 2013), mode suppression by amplitude death (Biwa, Tozuka \& Yazaki 2015) and analysis of the coupling between natural modes of similar frequencies (Acharya, Bothien \& Lieuwen 2018).

For open-loop control, several researchers - including Bellows et al. (2008) and Guan et al. (2019a) - have shown that the forced synchronization of periodic thermoacoustic oscillations can be qualitatively modelled with a forced VDP-type oscillator. As noted in $\S 1.2$, several key features, including the Arnold tongues and asynchronous quenching, can be accurately reproduced. In this study, we extend this low-order modelling approach to the forced synchronization of ergodic $\mathbb{T}_{1,2}^{2}$ quasiperiodic oscillations so as to better understand how to control them. The primary advantage of this phenomenological approach is that, unlike classical mechanistic approaches, it does not require detailed knowledge of the internal coupling between the flame and its surrounding acoustic modes (Pikovsky et al. 2003). Instead, the main thermoacoustic elements (flame and combustor) are lumped together and represented 
as a consolidated self-excited oscillator with discrete natural frequencies, which is forced externally at a different frequency. A secondary advantage of this approach is that nearly a century of knowledge and analytical solutions on classical oscillators, dating back to the work of van der Pol \& van der Mark (1927), can be repurposed for the control of modern systems such as combustors.

\subsection{Model development}

Our experiments show that the self-excited state of the system features ergodic $\mathbb{T}_{1,2}^{2}$ quasiperiodicity $(\$ 3.1)$. Therefore, we model the system as two autonomous coupled VDP oscillators with incommensurate natural frequencies subjected to external forcing:

$$
\left.\begin{array}{l}
\ddot{x}_{1}-b_{1} \dot{x}_{1}+\omega_{1}^{2} x_{1}+x_{1}^{2} \dot{x}_{1}+k\left(x_{1}-x_{2}\right)=A_{f} \sin \left(\omega_{f} t\right), \\
\ddot{x}_{2}-b_{2} \dot{x}_{2}+\omega_{2}^{2} x_{2}+x_{2}^{2} \dot{x}_{2}+k\left(x_{2}-x_{1}\right)=B_{f} \sin \left(\omega_{f} t\right),
\end{array}\right\}
$$

where $x_{1}$ and $x_{2}$ are the dynamical variables, $b_{1}$ and $b_{2}$ are the linear growth parameters for self-excitation, $\omega_{1}$ and $\omega_{2}$ are the natural angular frequencies and $k$ is the strength of the internal coupling between the two oscillators. The internal coupling is linear, reactive and symmetric, and saturation is provided by cubic nonlinearity. These choices are meant to keep the model as simple as possible, consistent with our phenomenological approach. The forcing is external and sinusoidal, with an angular frequency of $\omega_{f}$ and amplitudes of $A_{f}$ and $B_{f}$ for the two respective oscillators. For simplicity, the forcing is assumed to act on both oscillators equally $\left(A_{f}=B_{f}\right)$ and simultaneously, in accordance with the framework of Kashinath et al. (2018).

The model (4.1) is calibrated using only two ratios from the unforced experiments (§3.1): (i) the winding number, $f_{2} / f_{1}=0.70$, which is used to define $\omega_{2} / \omega_{1}=0.70$; and (ii) the spectral amplitude ratio for modes $f_{1} / f_{2}(\approx 10)$, which is used to define $b_{1}=0.04, b_{2}=0.018$ and $k=0.2$. Although the model is calibrated using quantitative ratios, its simple form implies that it remains purely phenomenological, providing no expectations of a quantitative match with the experimental results. The model is solved numerically using a multistage variable-order algorithm (Shampine \& Reichelt 1997). This is done for a range of $A_{f}$ and $\omega_{f}$ so as to reproduce the experimental conditions.

Although Anishchenko et al. (2008) have used a similar model to study the forced synchronization of quasiperiodic oscillations, they focused on a resonant limit cycle rather than an ergodic $\mathbb{T}_{1,2}^{2}$ torus $(\$ 1.3)$. In particular, they examined the dynamical states and bifurcations of the system, which are important for understanding its inherent stability. As our study is motivated by open-loop control, we build on the work of Anishchenko et al. (2008) by focusing on the partial and complete synchronization boundaries as well as on amplitude-related phenomena such as asynchronous quenching and resonant amplification.

\subsection{Comparison with experiments}

Figure 8 compares the synchronization maps of $(a) \eta_{p^{\prime}}$ from the experiments and (b) $\eta_{x^{\prime}}$ from the model. Here $\eta_{x^{\prime}}$ is defined analogously to $\eta_{p^{\prime}}$ but for $x^{\prime}(t)=x_{1}^{\prime}(t)$ instead of $p^{\prime}(t)$. As before, the response amplitude is weakened by the forcing when $\eta_{x^{\prime}}<0$ (blue regions) but is amplified by the forcing when $\eta_{x^{\prime}}>0$ (red regions). Overlaid on the contours of $\eta_{p^{\prime}}$ and $\eta_{x^{\prime}}$ are discrete markers representing the minimum forcing amplitude required for the synchronization of the weak natural mode $\left(f_{2} ; \omega_{2}\right)$, the strong natural mode $\left(f_{1} ; \omega_{1}\right)$ and both natural modes $\left(f_{1}, f_{2} ; \omega_{1}, \omega_{2}\right)$ - the latter corresponding to complete synchronization. The experimental map (figure $8 a$ ) is only 

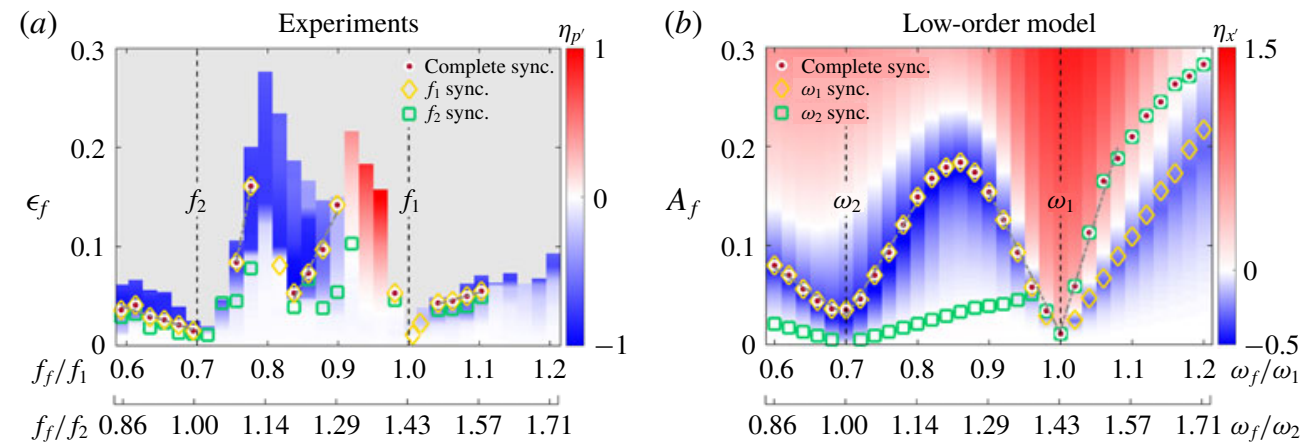

FIgURE 8. (Colour online) Synchronization maps from $(a)$ the experiments and $(b)$ the low-order model showing contours of the normalized response amplitude $\left(\eta_{p^{\prime}} ; \eta_{x^{\prime}}\right)$, in a parameter space defined by the forcing frequency and amplitude. Three sets of markers are shown, representing the minimum forcing amplitude required for the synchronization of the weak natural mode $\left(f_{2} ; \omega_{2}\right)$, the strong natural mode $\left(f_{1} ; \omega_{1}\right)$ and both natural modes $\left(f_{1}, f_{2} ; \omega_{1}, \omega_{2}\right)$ - the latter corresponding to complete synchronization. In $(a)$, the grey background regions denote FBO.

partially filled with data because of FBO (grey background regions), whereas the modelling map (figure $8 b$ ) has no such restrictions and can therefore offer predictive insight into the dynamics potentially exhibited by the thermoacoustic system had it not succumbed to FBO.

Despite its simplicity and trivial calibration, the model can still qualitatively reproduce many, but not all, of the synchronization features observed experimentally in the thermoacoustic system. These features include: (i) the emergence of twoand three-frequency quasiperiodic states as the forcing amplitude increases at an off-resonance frequency, as illustrated in figure 9: $\left(A_{f}=0\right)$ unforced ergodic $\mathbb{T}_{1,2}^{2}$ quasiperiodicity $\rightarrow\left(A_{f}=0.008\right)$ ergodic $\mathbb{T}_{1,2, f}^{3}$ quasiperiodicity $\rightarrow\left(A_{f}=0.042\right.$ and 0.052$)$ resonant $\mathbb{T}_{1, f}^{2}$ quasiperiodicity $\rightarrow\left(A_{f} \geqslant 0.068\right)$ synchronous $P 1_{f}$ periodicity; (ii) a sequential route to complete synchronization along which the weak natural mode $\left(f_{2} ; \omega_{2}\right)$ synchronizes before the strong natural mode $\left(f_{1} ; \omega_{1}\right)$, although the model reverses this ordering when $\omega_{f} / \omega_{1}>1$; (iii) a reduction in the minimum forcing amplitude required for partial and complete synchronization as the forcing frequency approaches either of the two natural frequencies, resulting in two primary Arnold tongues; (iv) a wide range of phase dynamics, including phase drifting, slipping, trapping and locking (figure 9c); (v) a reduction in the response amplitude due to asynchronous quenching for forcing frequencies away from the strong natural mode $\left(f_{1} ; \omega_{1}\right)$, with the maximum reduction typically occurring near complete synchronization (figure 10a); and (vi) an increase in the response amplitude due to resonant amplification for forcing frequencies close to the strong natural mode $\left(f_{1}\right.$; $\omega_{1}$ ), although the model shows some amplification even before the onset of complete synchronization when $\omega_{f}>\omega_{1}$ (figure $10 b$ ).

There are, however, several features of the thermoacoustic system that cannot be reproduced by the model. These features include: (i) the existence of a partial Arnold tongue between $f_{1}$ and $f_{2}$; (ii) the re-emergence of the $f_{1}$ and $f_{2}$ modes when $\epsilon_{f}$ increases above that required for complete synchronization, leading to transitions out of $P 1_{f}$ and into $\mathbb{T}_{1,2, f}^{3}$ or $\mathbb{T}_{2, f}^{2}$; and (iii) a reduction in the response amplitude at complete synchronization when $f_{f} / f_{1}>1$. 
(a)

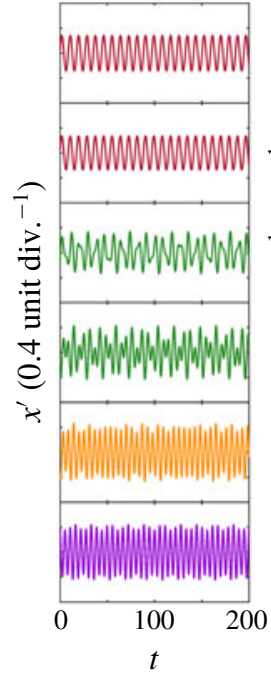

(b)

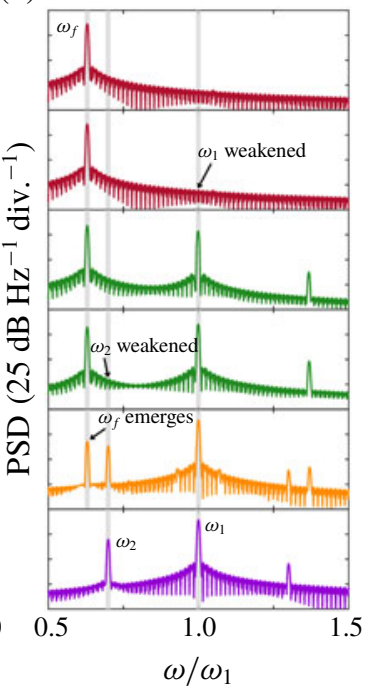

(c)

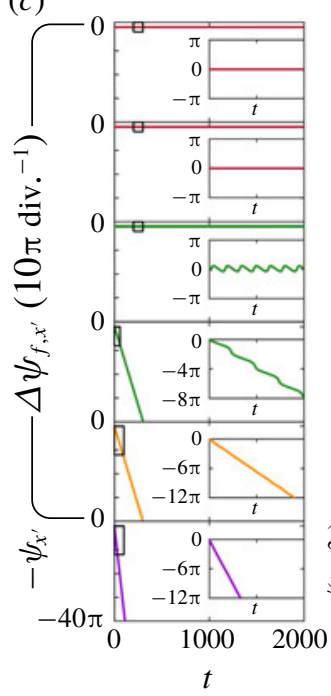

(d)

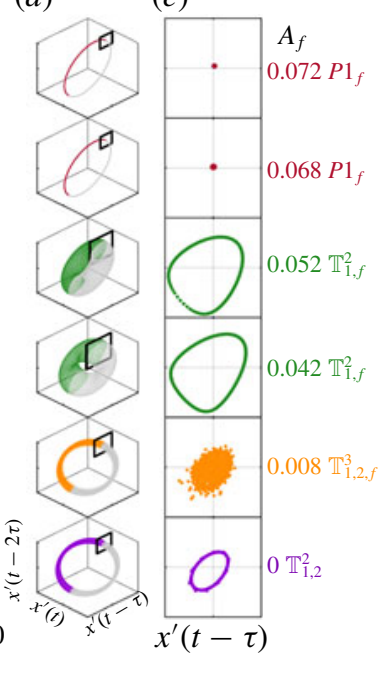

FIGURE 9. (Colour online) Forced response of two coupled VDP oscillators (4.1) with self-excited ergodic $\mathbb{T}_{1,2}^{2}$ quasiperiodicity undergoing asynchronous quenching at $\omega_{f} / \omega_{1}=$ $0.63\left(\omega_{f} / \omega_{2}=0.90\right)$ for six values of $A_{f}$, including the unforced case $\left(A_{f}=0\right)$ : $(a)$ time trace, $(b)$ PSD, $(c) \Delta \psi_{f, x^{\prime}}$ (bottom row: $-\psi_{x^{\prime}}$ ), $(d)$ phase portrait and $(e)$ Poincaré map. In $(d, e)$, phase-space reconstruction is performed with $d=3$ and $\tau=1.8$. The bottom panel of $(c)$ shows $-\psi_{x^{\prime}}$, rather than $\Delta \psi_{f, x^{\prime}}$, because $A_{f}=0$ there.

(a)

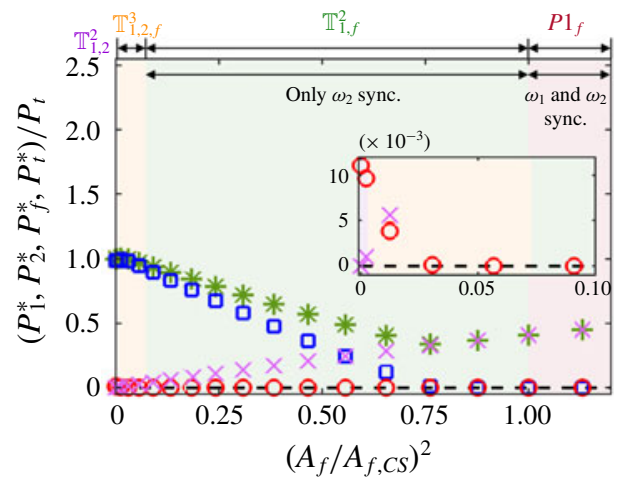

(b)

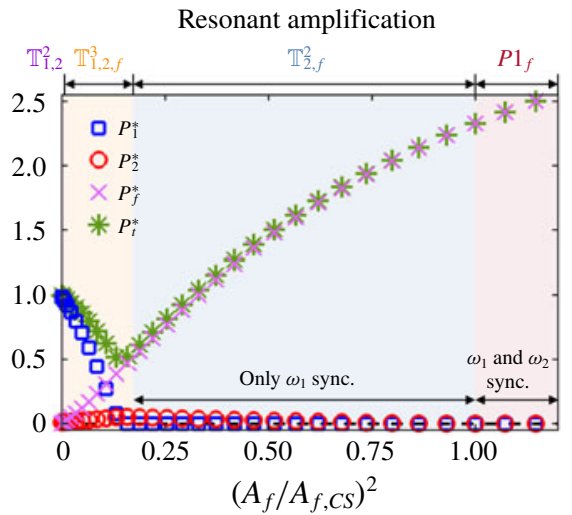

FIGURE 10. (Colour online) Representative cases of (a) asynchronous quenching and (b) resonant amplification in two forced coupled VDP oscillators (4.1) with self-excited ergodic $\mathbb{T}_{1,2}^{2}$ quasiperiodicity. The spectral powers of the $\omega_{1}, \omega_{2}$ and $\omega_{f}$ modes, along with the total power, are shown as a function of the forcing power normalized by that required to cause the onset of complete synchronization. The forcing frequencies are (a) $\omega_{f} / \omega_{1}=0.63$ or $\omega_{f} / \omega_{2}=0.90$ and $(b) \omega_{f} / \omega_{1}=1.04$ or $\omega_{f} / \omega_{2}=1.49$.

The double-Arnold-tongue behaviour seen in both the experiments (figure $8 a$ ) and the model (figure 8b) can be explained through the internal coupling between the two oscillators. Supplementary simulations of the model (not shown) indicate that, without 
internal coupling ( $k=0$ in (4.1)), the two oscillators are effectively independent: the synchronization boundary of the $\omega_{1}$ mode contains just one Arnold tongue centred on $\omega_{f} / \omega_{1}=1$, while that of the $\omega_{2}$ mode also contains just one Arnold tongue but centred on $\omega_{f} / \omega_{2}=1$. The fact that, in both the experiments (figure $8 a$ ) and the coupled model (figure $8 b$ ), we find two Arnold tongues in each of the two synchronization boundaries suggests that the forcing is being internally amplified: after the weaker natural mode is synchronized, it goes on to amplify the forcing signal such that the remaining natural mode becomes synchronized at a lower (external) forcing amplitude than would otherwise be required without the synchronization of the first natural mode. Such internal amplification of the forcing is consistent with the laser experiments of Loose et al. (2010), who showed that weak internal coupling leads to the partial synchronization of only one natural mode, but that strong internal coupling leads to the complete synchronization of both natural modes in succession. This concurs with the dynamics observed in our thermoacoustic system and in the coupled VDP oscillators.

\section{Conclusions}

In this study, we have taken a synchronization approach to answering three research questions on the open-loop control of ergodic $\mathbb{T}_{1,2}^{2}$ quasiperiodic oscillations in a prototypical thermoacoustic system - a laminar conical premixed flame in a tube combustor. The three questions (\$1.4) and our answers to them are as follows.

(i) Previous studies have shown that periodic acoustic forcing can control periodic thermoacoustic oscillations $(\S 1.2)$, but can it also control quasiperiodic thermoacoustic oscillations? If it can, how does the synchronization process differ from that of the classical period-1 case studied by Guan et al. (2019a) and Mondal et al. (2019)?

Using spectral analysis and nonlinear time-series analysis, we have provided experimental evidence showing that periodic acoustic forcing is an effective strategy for controlling ergodic $\mathbb{T}_{1,2}^{2}$ quasiperiodic thermoacoustic oscillations $(\S 3)$. Not only can the two natural frequencies $\left(f_{1}\right.$ and $\left.f_{2}\right)$ be shifted to the forcing frequency $\left(f_{f}\right)$ at a critically high forcing amplitude $\left(\epsilon_{f}\right)$, but the thermoacoustic amplitude $\left(\eta_{p^{\prime}}\right)$ can be simultaneously reduced, particularly when the system is completely synchronized. Compared with that of a classical period-1 system, complete synchronization of this ergodic $\mathbb{T}_{1,2}^{2}$ quasiperiodic system is found to occur via a more elaborate route involving three sequential steps: as $\epsilon_{f}$ increases, $(a)$ the system first transitions from unforced ergodic $\mathbb{T}_{1,2}^{2}$ quasiperiodicity to ergodic $\mathbb{T}_{1,2, f}^{3}$ quasiperiodicity owing to the introduction of an additional active DOF by the forcing $(\$ 3.2 .1)$; $(b)$ the system then transitions to resonant $\mathbb{T}_{1, f}^{2}$ quasiperiodicity as the weaker of the two natural modes $\left(f_{2}\right)$ synchronizes first, leading to partial synchronization (\$3.2.2); and finally $(c)$ the system transitions to a $P 1_{f}$ limit cycle as the remaining natural mode $\left(f_{1}\right)$ also synchronizes, leading to complete synchronization ( $\left.\$ 3.2 .3\right)$. The minimum $\epsilon_{f}$ required to cause partial and complete synchronization decreases as $f_{f}$ approaches either $f_{1}$ or $f_{2}$, resulting in two primary Arnold tongues, one centred on $f_{f} / f_{1}=1$ and the other on $f_{f} / f_{2}=1$. There is also a third Arnold tongue between $f_{1}$ and $f_{2}$, but it exists only partially $(\$ 3.2 .3)$. The two primary Arnold tongues, along with the three-step sequential route to complete synchronization, can be modelled with low-order universal oscillators $(\$ 1.3)$, but have been observed in only two experimental systems before, neither of which is fluid mechanical: electronic circuits (Anishchenko et al. 2007) and semiconductor lasers (Loose et al. 2010). Furthermore, when forced at an amplitude above that required for $P 1_{f}$, the system can transition 
out of this completely synchronous state and into other states (§3.2.4), including resonant $\mathbb{T}_{2, f}^{2}$ quasiperiodicity, ergodic $\mathbb{T}_{1,2, f}^{3}$ quasiperiodicity and flame blow-off (FBO). Such desynchronization to high-order quasiperiodic states at high $\epsilon_{f}$ is a possible precursor to chaos. Associated with these various nonlinear states is a wide range of phase dynamics, including phase drifting, slipping, trapping and locking. In summary, we have provided the first experimental demonstration of open-loop control on quasiperiodic thermoacoustic oscillations, extending the applicability of this active control strategy from classical period-1 systems with just a single time scale to ergodic $\mathbb{T}^{2}$ quasiperiodic systems with two incommensurate time scales.

(ii) If periodic acoustic forcing can control quasiperiodic thermoacoustic oscillations, what are the optimal forcing conditions for producing the maximum reduction in thermoacoustic amplitude?

By analysing the contours of $\eta_{p^{\prime}}$ and the spectral powers of the $f_{1}, f_{2}$ and $f_{f}$ modes, we have experimentally shown that the optimal control strategy is to apply off-resonance forcing at a frequency around the weaker natural mode $\left(f_{2}\right)$ and at an amplitude just sufficient to cause complete synchronization $\left(P 1_{f}\right)$, as this produces the largest reduction in $\eta_{p^{\prime}}$ using the least actuation effort by exploiting internal coupling ( $\$ 3.2 .5$ and 4). The reduction in $\eta_{p^{\prime}}$ occurs via asynchronous quenching of the two natural modes, which, according to the Rayleigh index, is physically caused by a disruption of the positive feedback between the unsteady HRR of the flame and the acoustic modes of the combustor $(\$ 3.3)$. For reliable control, care must be taken to avoid forcing the system near its dominant natural mode $\left(f_{1}\right)$ because here $\eta_{p^{\prime}}$ and the Rayleigh index can increase with $\epsilon_{f}$ via resonant amplification of the forcing signal (\$3.3). This criterion for resonant amplification is also valid for a classical period-1 system (Guan et al. 2019a). Thus, this demonstrates that the addition of a weak natural mode $\left(f_{2}\right)$ to a single-mode $\left(f_{1}\right)$ system can make open-loop control easier to implement, as the forcing can now be applied around $f_{2}$, instead of $f_{1}$, with similar reductions in $\eta_{p^{\prime}}$ but less risk of resonant amplification.

However, it should be cautioned that this control strategy only works if one of the natural modes is significantly weaker than the other. If both modes are of similar strength, then it is conceivable that strong resonant amplification of the forcing can occur near both modes. In fact, this is exactly the outcome predicted by coupled VDP simulations. Therefore, when both natural modes are similar in strength, the proposed control strategy may have to be modified such that forcing is applied at a frequency away from both $f_{1}$ and $f_{2}$, so as to avoid regimes of resonant amplification.

(iii) Previous studies on electronic circuits and semiconductor lasers have shown that the forced synchronization of quasiperiodic oscillations can be qualitatively modelled with low-order universal oscillators containing a VDP kernel (§1.3), but can such a phenomenological modelling approach work on thermoacoustic systems as well?

We have shown that a pair of reactively coupled VDP oscillators subjected to external sinusoidal forcing can qualitatively reproduce many, but not all, of the synchronization features of the thermoacoustic system described above. The only features that cannot be reproduced are $(a)$ the partial Arnold tongue between $f_{1}$ and $f_{2} ;(b)$ the re-emergence of the $f_{1}$ and $f_{2}$ modes when $\epsilon_{f}$ increases above that required for complete synchronization, leading to transitions out of $P 1_{f}$ and into $\mathbb{T}_{1,2, f}^{3}$ or $\mathbb{T}_{2, f}^{2}$; and $(c)$ asynchronous quenching at $P 1_{f}$ when $f_{f} / f_{1}>1$. Nevertheless, it is worth recalling that this model is the simplest self-excited model with two incommensurate natural modes. Despite its simplicity and trivial calibration, it can still qualitatively reproduce the synchronization features pertinent to open-loop control, such as the partial/complete synchronization boundaries, the two primary Arnold 
tongues, asynchronous quenching and resonant amplification. This phenomenological agreement between a prototypical thermoacoustic system and a canonical model shows that these synchronization features are not specific to our particular system, but are universal features of forced self-excited oscillators.

The ability to model a real thermoacoustic system as a low-order oscillator system opens up new possibilities for the development of alternative control strategies, as the equations used to build the low-order system (nonlinear ordinary differential equations with just a few DOFs) are much simpler than the full Navier-Stokes equations (nonlinear partial differential equations with infinite DOFs) and can therefore be solved more easily. However, it should be cautioned that before such a model can be of practical use, its nonlinear saturation and coupling terms must be determined more accurately. If this can be achieved, the analytical and numerical solutions provided by such a model can be used for various applications, including: $(a)$ as a prototypical platform for testing passive and active control strategies; $(b)$ to gain new insight into the physics of multi-mode thermoacoustic oscillations and their mutual synchronization; $(c)$ to investigate the interactions between thermoacoustic oscillations in the combustor and flow oscillations in other sub-systems, such as Helmholtz resonators; $(d)$ to explore how the stability boundaries are influenced by different types of noise; and $(e)$ to guide the reduction and analysis of experimental or numerical data collected in real combustors, with the aim of identifying subtle dynamics that might otherwise be overlooked.

Regarding opportunities for future work, we note that an effect worth exploring is that of the speed with which the forcing conditions are varied. Recent thermoacoustics experiments by Bonciolini \& Noiray (2019) have shown that it is possible to dodge a supercritical Hopf bifurcation if the bifurcation parameter can be varied sufficiently quickly. It is thus reasonable to expect an analogous phenomenon occurring in forced synchronization, whereby it may be possible to dodge certain $\mathbb{T}^{2}$ or $\mathbb{T}^{3}$ states en route to complete synchronization if $f_{f}$ or $\epsilon_{f}$ can be varied sufficiently quickly. A full investigation of such a transient effect is best left for a future study.

Another effect worth exploring is that of turbulence. Although our findings are qualitatively reproducible across a range of forcing and operating conditions, they have only been demonstrated here on a prototypical laminar combustor, with none of the extra complexity, bifurcations and DOFs that turbulence brings. Turbulence, and noise in general, can have non-trivial effects on the stability and dynamics of a nonlinear system, because it can modify both the deterministic properties (Noiray \& Schuermans 2013) and the stochastic properties (Kabiraj et al. 2015; Gopalakrishnan et al. 2016). Nevertheless, experiments by Bellows et al. (2008) and Balusamy et al. (2015) on turbulent combustors undergoing self-excited periodic oscillations have shown that many of the defining features of forced synchronization can still be recovered. Moreover, decades of experiments, simulations and theoretical analyses in nonlinear dynamics have established that such synchronization features often arise across a variety of physically disparate systems, ranging from respiratory rhythms to neural cells to triode circuits (Pikovsky et al. 2003; Balanov et al. 2008). Our next step is to conduct experiments under increasingly turbulent conditions to determine the extent to which the present findings carry over into practical systems.

\section{Acknowledgements}

We would like to thank Professor M. Juniper for introducing us to this problem, and Dr K. Kashinath for patiently helping us understand the results of his PhD thesis. 


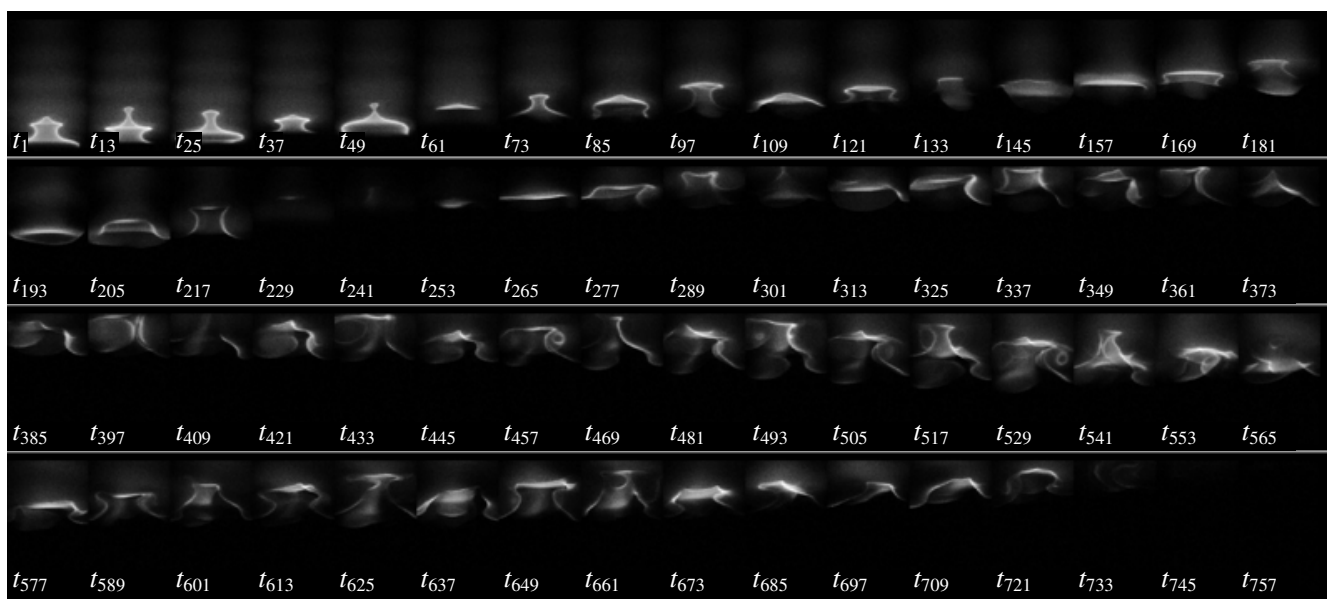

FIGURE 11. A sequence of time-resolved flame images captured via broadband chemiluminescence during an FBO event. Successive images are separated in time by $4 \mathrm{~ms}$. The forcing conditions are $f_{f} / f_{1}=0.84, f_{f} / f_{2}=1.20$, and $\epsilon_{f}=0.19$.

This work was supported by the Research Grants Council of Hong Kong (Project nos 16235716, 26202815 and 16210418), the National Natural Science Foundation of China (grant nos 11672123 and 91752201) and the Shenzhen Science and Technology Program (grant no. JCYJ20170412151759222).

\section{Appendix A. Flame dynamics near blow-off conditions}

Figure 11 shows a sequence of time-resolved flame images captured via broadband chemiluminescence during an FBO event. In the early stages of FBO ( $t_{1}$ to $\left.t_{49}\right)$, the flame base lifts off aperiodically from the burner lip as a result of strong external forcing. Roll-up wrinkles can be seen advecting along the flame body, producing axisymmetric perturbations as well as pinch-off at the flame tip ( $t_{13}$ and $\left.t_{49}\right)$. Subsequently $\left(t>t_{49}\right)$, the flame base detaches permanently from the burner lip, causing the flame body to move around chaotically with occasional transient near-extinction events $\left(t_{241}\right)$. As time evolves, the flame attempts to reattach itself to the burner lip ( $t_{385}$ to $\left.t_{565}\right)$ but fails and extinguishes, completing the FBO event $\left(t_{733}\right)$.

\section{Appendix B. Transient response en route to asynchronous quenching}

To illustrate the transient response of the system en route to asynchronous quenching, we show in figure 12 time traces of the normalized $p^{\prime}(t)$ signal and $\epsilon_{f}(t)$ at $f_{f} / f_{1}=0.63\left(f_{f} / f_{2}=0.90\right)$. These forcing conditions correspond to those of figure 4 (third row from the top). It can be seen that, after the forcing is activated, the system takes around $2 \mathrm{~s}$ to transition from $\mathbb{T}_{1,2}^{2}$ to $P 1_{f}$. However, the accompanying reduction in thermoacoustic amplitude $\left(\eta_{p^{\prime}}=-0.87\right)$ due to asynchronous quenching occurs earlier, taking just around $0.5 \mathrm{~s}$. After the forcing is deactivated, the system takes around $0.5 \mathrm{~s}$ to transition back from $P 1_{f}$ to $\mathbb{T}_{1,2}^{2}$ as well as to return to its original high-amplitude oscillatory state. This demonstrates that this open-loop control strategy, when applied to the prototypical quasiperiodic thermoacoustic system studied here, is reasonably quick and can be considered for further testing on more realistic combustors. 


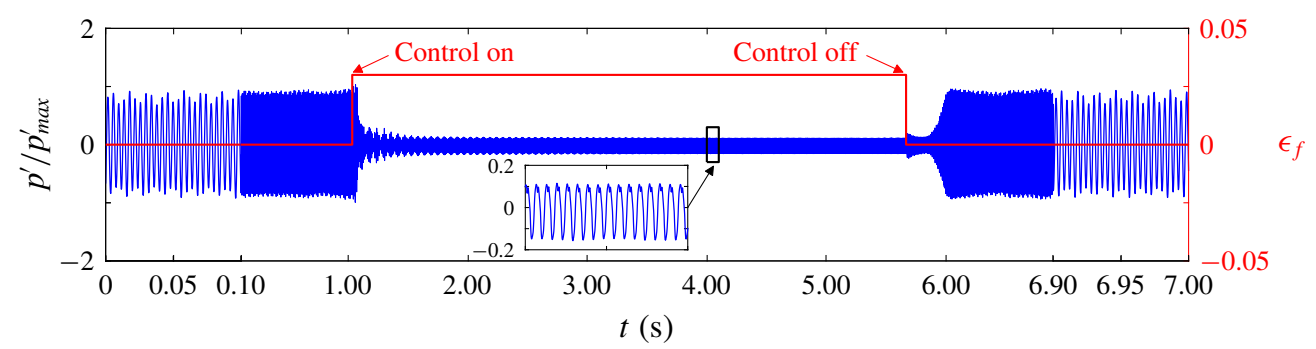

FIgURE 12. (Colour online) Transient response of an ergodic $\mathbb{T}_{1,2}^{2}$ thermoacoustic system undergoing asynchronous quenching via the open-loop application of periodic acoustic forcing at $f_{f} / f_{1}=0.63\left(f_{f} / f_{2}=0.90\right)$. The reduction in thermoacoustic amplitude is $\eta_{p^{\prime}}=$ -0.87 , which is achieved with an actuator power of just $0.001 \%$ of the thermal power of the flame.

\section{REFERENCES}

Acharya, V. S., Bothien, M. R. \& Lieuwen, T. C. 2018 Non-linear dynamics of thermoacoustic eigen-mode interactions. Combust. Flame 194, 309-321.

Afraimovich, V. S. \& Shilnikov, L. P. 1991 Invariant two-dimensional tori, their breakdown and stochasticity. Am. Math. Soc. Transl. 149 (2), 201-212.

Anishchenko, V., Nikolaev, S. \& KURThs, J. 2007 Peculiarities of synchronization of a resonant limit cycle on a two-dimensional torus. Phys. Rev. E 76 (4), 046216.

Anishchenko, V., Nikolaev, S. \& KURThS, J. 2008 Bifurcational mechanisms of synchronization of a resonant limit cycle on a two-dimensional torus. Chaos 18 (3), 037123.

Balanov, A., Janson, N., Postnov, D. \& Sosnovtseva, O. 2008 Synchronization: From Simple to Complex. Springer Science and Business Media.

Balusamy, S., Li, L. K. B., Han, Z., Juniper, M. P. \& Hochgreb, S. 2015 Nonlinear dynamics of a self-excited thermoacoustic system subjected to acoustic forcing. Proc. Combust. Inst. 35 (3), 3229-3236.

Battelino, P. M. 1988 Persistence of three-frequency quasiperiodicity under large perturbations. Phys. Rev. A 38 (3), 1495-1502.

Bellows, B., Hreiz, A.\& Lieuwen, T. 2008 Nonlinear interactions between forced and self-excited acoustic oscillations in premixed combustor. J. Propul. Power 24 (3), 628-631.

Biwa, T., TozUKa, S. \& YAZAKI, T. 2015 Amplitude death in coupled thermoacoustic oscillators. Phys. Rev. Appl. 3 (3), 034006.

Bonshash, B. 1992 Estimating and interpreting the instantaneous frequency of a signal. Proc. IEEE 80 (4), 520-538.

Boccaletti, S., Allaria, E., Meucci, R. \& Arecchi, F. T. 2002 Experimental characterization of the transition to phase synchronization of chaotic $\mathrm{CO}_{2}$ laser systems. Phys. Rev. Lett. 89 (19), 194101.

BONCIOLINI, G. \& NOIRAY, N. 2019 Bifurcation dodge: avoidance of a thermoacoustic instability under transient operation. Nonlinear Dyn. 1, 1-14.

Borkowski, L., Perlikowski, P., Kapitaniak, T. \& Stefanski, A. 2015 Experimental observation of three-frequency quasiperiodic solution in a ring of unidirectionally coupled oscillators. Phys. Rev. E 91 (6), 062906.

Bothien, M. R., Moeck, J. P. \& Paschereit, C. O. 2008 Active control of the acoustic boundary conditions of combustion test rigs. J. Sound Vib. 318 (4), 678-701.

Bourehla, A. \& BAillot, F. 1998 Appearance and stability of a laminar conical premixed flame subjected to an acoustic perturbation. Combust. Flame 114 (3), 303-318.

CAndel, S. 2002 Combustion dynamics and control: progress and challenges. Proc. Combust. Inst. 29 (1), 1-28. 
CAO, L. 1997 Practical method for determining the minimum embedding dimension of a scalar time series. Physica D 110 (1), 43-50.

CUlick, F. E. C. 1971 Non-linear growth and limiting amplitude of acoustic oscillations in combustion chambers. Combust. Sci. Technol. 3 (1), 1-16.

Culick, F. E. C. 2006 Unsteady motions in combustion chambers for propulsion systems. North Atlantic Treaty Organisation, AGARDograph AG-AVT-039.

Davitian, J., Getsinger, D., Hendrickson, C. \& Karagozian, A. R. 2010 Transition to global instability in transverse-jet shear layers. J. Fluid Mech. 661, 294-315.

DewAN, E. 1972 Harmonic entrainment of van der Pol oscillations: phaselocking and asynchronous quenching. IEEE Trans. Autom. Control 17 (5), 655-663.

Dowling, A. P. \& Morgans, A. S. 2005 Feedback control of combustion oscillations. Annu. Rev. Fluid Mech. 37, 151-182.

FraSER, A. M. \& SWINNEY, H. L. 1986 Independent coordinates for strange attractors from mutual information. Phys. Rev. A 33 (2), 1134-1140.

GABOR, D. 1946 Theory of communication. J. Inst. Electr. Engng (London) 3, 429-457.

GAYDON, A. G. 1974 Spectroscopy of Flames. Chapman and Hall.

GLASS, L. 2001 Synchronization and rhythmic processes in physiology. Nature 410 (6825), 277-284.

Gollub, J. P. \& Benson, S. V. 1980 Many routes to turbulent convection. J. Fluid Mech. 100 (3), 449-470.

Gopalakrishnan, E. A., Tony, J., Sreelekha, E. \& Sujith, R. I. 2016 Stochastic bifurcations in a prototypical thermoacoustic system. Phys. Rev. E 94 (2), 022203.

Gotoda, H., OkUno, Y., Hayashi, K. \& Tachibana, S. 2015 Characterization of degeneration process in combustion instability based on dynamical systems theory. Phys. Rev. E 92 (5), 052906.

Gottwald, G. A. \& Melbourne, I. 2004 A new test for chaos in deterministic systems. Proc. R. Soc. Lond. A 460 (2042), 603-611.

Grassberger, P. \& Procaccia, I. 1983 Characterization of strange attractors. Phys. Rev. Lett. 50 (5), 346-349.

Guan, Y., Gupta, V., Kashinath, K. \& Li, L. K. B. $2019 a$ Open-loop control of periodic thermoacoustic oscillations: experiments and low-order modelling in a synchronization framework. Proc. Combust. Inst. 37, 5315-5323.

Guan, Y., He, W., Murugesan, M., LI, Q., Liu, P. \& Li, L. K. B. $2019 b$ Control of self-excited thermoacoustic oscillations using transient forcing, hysteresis and mode switching. Combust. Flame 202, 262-275.

Guan, Y., Murugesan, M. \& LI, L. K. B. 2018 Strange nonchaotic and chaotic attractors in a self-excited thermoacoustic oscillator subjected to external periodic forcing. Chaos 28 (9), 093109.

Gustafsson, F. 1996 Determining the initial states in forward-backward filtering. IEEE Trans. Signal Process. 44 (4), 988-992.

Heckl, M. A. 1988 Active control of the noise from a Rijke tube. J. Sound Vib. 124 (1), 117-133.

Hilborn, R. C. 2000 Chaos and Nonlinear Dynamics: An Introduction for Scientists and Engineers. Oxford University Press.

Hong, S., Shanbhogue, S. J., Speth, R. L. \& Ghoniem, A. F. 2013 On the phase between pressure and heat release fluctuations for propane/hydrogen flames and its role in mode transitions. Combust. Flame 160 (12), 2827-2842.

Hovel, P. 2010 Control of Complex Nonlinear Systems with Delay. Springer.

JAhnke, C. C. \& Culick, F. E. C. 1994 Application of dynamical systems theory to nonlinear combustion instabilities. J. Propul. Power 10 (4), 508-517.

Johnson, A., Uddin, M. \& Pollard, A. 2005 Calibration of hot-wire probes using non-uniform mean velocity profiles. Exp. Fluids 39 (3), 525-532.

JUNiPER, M. P. \& SUJith, R. I. 2018 Sensitivity and nonlinearity of thermoacoustic oscillations. Annu. Rev. Fluid Mech. 50, 661-689.

Kabiraj, L., Saurabh, A., Wahi, P. \& Sujith, R. I. $2012 a$ Route to chaos for combustion instability in ducted laminar premixed flames. Chaos 22 (2), 023129. 
Kabiraj, L., Steinert, R., Saurabh, A. \& Paschereit, C. O. 2015 Coherence resonance in a thermoacoustic system. Phys. Rev. E 92 (4), 042909.

Kabiraj, L. \& Sujith, R. I. 2012 Nonlinear self-excited thermoacoustic oscillations: intermittency and flame blowout. J. Fluid Mech. 713, 376-397.

Kabiraj, L., Sujith, R. I. \& Wahi, P. $2012 b$ Bifurcations of self-excited ducted laminar premixed flames. Trans. ASME: J. Engng Gas Turbines Power 134 (3), 031502.

Kantz, H. \& Schreiber, T. 2003 Nonlinear Time Series Analysis, 2nd edn. Cambridge University Press.

KAShinath, K., LI, L. K. B. \& JUniper, M. P. 2018 Forced synchronization of periodic and aperiodic thermoacoustic oscillations: lock-in, bifurcations and open-loop control. J. Fluid Mech. 838, 690-714.

Kashinath, K., Waugh, I. C. \& JUniper, M. P. 2014 Nonlinear self-excited thermoacoustic oscillations of a ducted premixed flame: bifurcations and routes to chaos. J. Fluid Mech. 761, 399-430.

Keen, B. E. \& Fletcher, W. H. W. 1970 Suppression of a plasma instability by the method of 'asynchronous quenching'. Phys. Rev. Lett. 24 (4), 130.

Khalak, A.\& Williamson, C. H. K. 1999 Motions, forces and mode transitions in vortex-induced vibrations at low mass-damping. J. Fluid Struct. 13, 813-851.

KulP, C. W. \& Zunino, L. 2014 Discriminating chaotic and stochastic dynamics through the permutation spectrum test. Chaos 24 (3), 033116.

LI, L. K. B. \& JUNIPER, M. P. $2013 a$ Lock-in and quasiperiodicity in a forced hydrodynamically self-excited jet. J. Fluid Mech. 726, 624-655.

LI, L. K. B. \& JUNIPER, M. P. $2013 b$ Lock-in and quasiperiodicity in hydrodynamically self-excited flames: experiments and modelling. Proc. Combust. Inst. 34 (1), 947-954.

LI, L. K. B. \& JUNIPER, M. P. $2013 c$ Phase trapping and slipping in a forced hydrodynamically self-excited jet. J. Fluid Mech. 735, R5.

Libchaber, A. \& MAURer, J. 1982 A Rayleigh Bénard experiment: helium in a small box. In Nonlinear Phenomena at Phase Transitions and Instabilities, pp. 259-286. Springer.

Lieuwen, T. C. 2003 Combustion driven oscillations in gas turbines. In Turbomachinery International, pp. 16-18. Turbomachinery International Publications.

Lieuwen, T. C. \& YAnG, V. 2005 Combustion Instabilities in Gas Turbine Engines: Operational Experience, Fundamental Mechanisms, and Modeling. American Institute of Aeronautics and Astronautics.

Loose, A., WÜNSChe, H. J. \& HenNeberger, F. 2010 Synchronization of quasiperiodic oscillations to a periodic force studied with semiconductor lasers. Phys. Rev. E 82 (3), 035201.

Lubarsky, E., Shcherbik, D., Bibik, A. \& Zinn, B. T. 2003 Active control of combustion oscillations by non-coherent fuel flow modulation. In Ninth AIAA/CEAS Aeroacoustics Conference and Exhibit, AIAA Paper, p. 3180.

Martin, S., Leber, H. \& Martienssen, W. 1984 Oscillatory and chaotic states of the electrical conduction in barium sodium niobate crystals. Phys. Rev. Lett. 53 (4), 303.

McManus, K. R., Vandsburger, U.\& Bowman, C. T. 1990 Combustor performance enhancement through direct shear layer excitation. Combust. Flame 82 (1), 75-92.

Minorsky, N. 1967 Comments on asynchronous quenching. IEEE Trans. Autom. Control 12 (2), 225-227.

Moeck, J. P. \& Paschereit, C. O. 2012 Nonlinear interactions of multiple linearly unstable thermoacoustic modes. Intl J. Spray Combust. 4 (1), 1-27.

Mondal, S., Pawar, S. A. \& Sujith, R. I. 2017 Synchronous behaviour of two interacting oscillatory systems undergoing quasiperiodic route to chaos. Chaos 27 (10), 103119.

Mondal, S., Pawar, S. A. \& SuJith, R. I. 2019 Forced synchronization and asynchronous quenching of periodic oscillations in a thermoacoustic system. J. Fluid Mech. 864, 73-96.

Mongia, H. C., Held, T. J., Hsiao, G. C. \& Pandalai, R. P. 2003 Challenges and progress in controlling dynamics in gas turbine combustors. J. Propul. Power 19 (5), 822-829.

Newhouse, S., Ruelle, D. \& TAKens, F. 1978 Occurrence of strange Axiom A attractors near quasiperiodic flows on $t^{m}, m \geqslant 3$. Commun. Math. Phys. 64 (1), 35-40. 
Noiray, N., Durox, D., Schuller, T. \& Candel, S. 2009 Dynamic phase converter for passive control of combustion instabilities. Proc. Combust. Inst. 32 (2), 3163-3170.

NolRay, N. \& Schuermans, B. 2013 Deterministic quantities characterizing noise driven Hopf bifurcations in gas turbine combustors. Intl J. Nonlinear Mech. 50, 152-163.

Odajima, K., Nishida, Y. \& Hatta, Y. 1974 Synchronous quenching of drift-wave instability. Phys. Fluids 17 (8), 1631-1633.

OrChini, A. \& JUniper, M. P. 2016 Flame double input describing function analysis. Combust. Flame 171, 87-102.

Pawar, S. A., Sujith, R. I., Emerson, B. \& Lieuwen, T. 2018 Characterization of forced response of density stratified reacting wake. Chaos 28 (2), 023108.

Pikovsky, A., Rosenblum, M. \& Kurths, J. 2003 Synchronization: A Universal Concept in Nonlinear Sciences. Cambridge University Press.

Pikovsky, A., Rosenblum, M., Osipov, G. \& Kurths, J. 1997 Phase synchronization of chaotic oscillators by external driving. Physica D 104 (3-4), 219-238.

Poinsot, T. 2017 Prediction and control of combustion instabilities in real engines. Proc. Combust. Inst. 36 (1), 1-28.

VAN DER POL, B. 1927 Forced oscillations in a circuit with non-linear resistance. Phil. Mag. 3 (13), 65-80.

VAN DER POL, B. \& VAN DER MARK, J. 1927 Frequency demultiplication. Nature 120 (3019), 363-364.

Provansal, M., Mathis, C. \& Boyer, L. 1987 Bénard-von Kármán instability: transient and forced regimes. J. Fluid Mech. 182, 1-22.

RAYLEIGH, LORD 1878 The explanation of certain acoustical phenomena. Nature 18, 319-321.

Ruelle, D. \& Takens, F. 1971 On the nature of turbulence. Commun. Math. Phys. 20 (3), 167-192.

Schmid, P. J. \& Henningson, D. S. 2012 Stability and Transition in Shear Flows. Springer Science and Business Media.

Shampine, L. F. \& Reichelt, M. W. 1997 The Matlab ODE suite. SIAM J. Sci. Comput. 18 (1), $1-22$.

Small, M. 2005 Applied Nonlinear Time Series Analysis: Applications in Physics, Physiology and Finance. World Scientific.

Stankevich, N. V., Kurths, J. \& Kuznetsov, A. P. 2015 Forced synchronization of quasiperiodic oscillations. Commun. Nonlinear Sci. 20 (1), 316-323.

Staubli, T. 1987 Entrainment of self-sustained flow oscillations: phaselocking or asynchronous quenching? J. Appl. Mech. 54, 707.

TAKens, F. 1981 Detecting strange attractors in turbulence. In Dynamical Systems and Turbulence (ed. D. A. Rand \& L. S. Young), Lecture Notes in Mathematics, pp. 366-381. Springer.

Thévenin, J., Romanelli, M., Vallet, M., Brunel, M. \& ErneuX, T. 2011 Resonance assisted synchronization of coupled oscillators: frequency locking without phase locking. Phys. Rev. Lett. 107 (10), 104101.

Thompson, J. M. T. \& Stewart, H. B. 2002 Nonlinear Dynamics and Chaos. John Wiley.

VAN BUSKIRK, R. \& JEFFRIES, C. 1985 Observation of chaotic dynamics of coupled nonlinear oscillators. Phys. Rev. A 31 (5), 3332.

Vishnu, R., Sujith, R. I. \& Aghalayam, P. 2015 Role of flame dynamics on the bifurcation characteristics of a ducted V-flame. Combust. Sci. Technol. 187 (6), 894-905.

Walden, R. W., Kolodner, P., Passner, A. \& Surko, C. M. 1984 Nonchaotic Rayleigh-Bénard convection with four and five incommensurate frequencies. Phys. Rev. Lett. 53 (3), 242.

Welch, P. D. 1967 The use of fast Fourier transform for the estimation of power spectra: a method based on time averaging over short modified periodograms. IEEE Trans. Audio Electroacoust. 15, 70-73. 